\title{
A Novel Energy-Saving Resource Allocation Scheme in LTE-A Relay Networks
}

\author{
Jen-Jee Chen, Chi-Wen Luo, and Zeng-Yu Chen \\ Department of Electrical Engineering, National University of Tainan, Tainan 70005, Taiwan \\ Correspondence should be addressed to Jen-Jee Chen; jjchen@mail.nutn.edu.tw
}

Received 21 December 2015; Revised 25 June 2016; Accepted 3 July 2016

Academic Editor: Gabriel-Miro Muntean

Copyright ( 2016 Jen-Jee Chen et al. This is an open access article distributed under the Creative Commons Attribution License, which permits unrestricted use, distribution, and reproduction in any medium, provided the original work is properly cited.

\begin{abstract}
The relay node (RN) in Long-Term Evolution-Advanced (LTE-A) networks is used to enhance the coverage of high data rate and solve the coverage hole problem. Considering the limited energy nature of User Equipment (UE), connecting to the RN instead of Evolved Node B (eNB) is a better choice for cell-edge UE items. In this paper, on the premise of compatibility to the LTE-A resource allocation specification, we discuss an uplink radio resource, uplink path, modulation and coding scheme (MCS), and transmit power allocation problem for energy conservation in LTE-A relay networks. The objective is to minimize the total energy consumption of UE items while guaranteeing the constraints of UE items' quality of service (QoS), bit-error-rate (BER), total system resource, and maximum transmit power. Since the problem is NP-complete and the scheduling period in LTE-A is short (the subframe length is only $1 \mathrm{~ms}$ ), we propose an efficient method to solve the problem. The complexity analysis shows the time complexity of the proposed heuristics is $O\left(n^{2}\right)$. Simulation results demonstrate that our algorithm can effectively reduce the energy consumption of UE items and guarantee users' service quality.
\end{abstract}

\section{Introduction}

In recent years, the third-generation partnership project (3GPP) has proposed the Long-Term Evolution (LTE) [1] and LTE-Advanced (LTE-A) [2] to support mobile and broadband wireless access in cellular systems. In LTE/LTE-A, the Orthogonal Frequency Division Multiple Access (OFDMA) is selected as the downlink access technology, which provides high spectrum efficiency, while, in the uplink, the SingleCarrier Frequency Division Multiple Access (SC-FDMA) technique is employed to reduce the Peak-to-Average Power Ratio (PAPR). Relay is one of the key features in LTE-A [3], where relays can enhance the coverage of high data rates, increase the throughput of cell-edge users, solve the coverage hole problem, and raise bandwidth utilization by spatial reuse. Two types of relays are introduced in the LTE-A. Type I relays act like eNBs to the attached UE items and have their own physical identities. On the contrary, Type II relays are transparent to the UE items and do not have physical identities. Like most wireless networks, energy saving is always an important issue for UE items due to the battery capacity restriction. Deploying relays, cell-edge UE items are able to save more power by connecting to the eNB via relays.

In this paper, we study the fundamental energy conservation problem in LTE-A uplink with Type I relays. We consider an uplink resource, path, MCS, and power allocation problem. The objective is to minimize the total energy consumption of UE items, while guaranteeing their constraints of relay network frame structure, maximum transmit power, BER, and QoS. Low power consumption is particularly important for UE items' batteries which can extend their lifetime. Today's wireless networks are characterized by a fixed spectrum assignment policy. Reference [4] shows that the average around $60 \%$ of the spectrum remains unutilized. This motivates us to exploit the idle spectrum to decrease the power consumption of UE items and thus increase UE items' battery lifetime and the spectrum utilization.

In the literature, much work has been done for the uplink resource allocation in LTE/LTE-A networks. Reference [5] proposes the optimal SC-FDMA resource allocation algorithm based on a pure binary-integer program to maximize the total user-weighted system capacity. Reference [6] 
presents a set of resource allocation schemes for LTE uplink to achieve the proportional fairness of users while maintaining good system throughput. However, the above studies $[5,6]$ do not take relays into consideration. For Type I relay networks, $[7,8]$ show how to achieve a good trade-off between system throughput and global proportional fairness over in-band and out-band relay networks, respectively. But, both of them focus on the downlink resource allocation and energy conservation is not the concern. In IEEE 802.16, [9] defines a resource allocation problem which aims at the minimization of energy consumption of UE items. The authors discuss the relationship between the MCSs and the energy consumption of UE. The result shows that the UE can decrease (resp., increase) its power consumption by choosing a lower (resp., higher) level of MCS but spend more (resp., less) physical resources. Reference [10] continues and extends the energyconserved resource allocation problem in IEEE 802.16j. However, both studies $[9,10]$ are not valid for LTE/LTE-A. Reference [11] examines the effect of Physical Resource Block (PRB) allocation on LTE UE's uplink transmission power and energy consumption. Simulation results show that, for each subframe, to allocate as many PRBs as possible to a single user is more energy efficient than sharing PRBs among several users. In [12], to improve the energy efficiency, user terminals cooperate with each other in transmitting their data packets to the base station (BS) by exploiting multitypes of wireless interfaces. To be specific, when two UE items are close to each other, they first exchange their data with short range communication interfaces. Once the negotiation is done, they share the antennas to transmit their data to BS by employing distributed space-time coding. Reference [13] proposes two power-efficient resource schedulers for LTE uplink systems subject to rate, delay, contiguous allocation, and maximum transmit power constraints. Reference [14] proposes a green opportunistic and efficient Resource Block (RB) allocation algorithm for LTE uplink networks, which maximizes the system throughput in an energy efficient way subject to users' QoS requirements and SC-FDMA constraints. Reference [15] proposes an energy efficient Medium Access Control (MAC) scheme for multiuser LTE downlink transmission, which utilizes the multiuser gain of the MIMO channel and the multiplexing gain of the multibeam opportunistic beamforming technique. Reference [16] discusses the buffer-overflow and buffer-underflow problems in the LTE-A relay network and presents adynamic flow control method to minimize the buffer-overflow and buffer-underflow probabilities. Reference [17] discusses the relay selection, power allocation, and subcarrier assignment problem and proposes a twolevel dual decomposition and subgradient method and two low-complexity suboptimal schemes to maximize the system throughput. Reference [18] examines the weighted power minimization problem and jointly optimizes the bandwidth and power usage under constraints on required rate, bandwidth, and transmit power. So far, there is no existing work studying the LTE-A relay network uplink energy conservation issue by considering the uplink path determination, radio resource scheduling, and MCS and transmit power allocation at the same time.

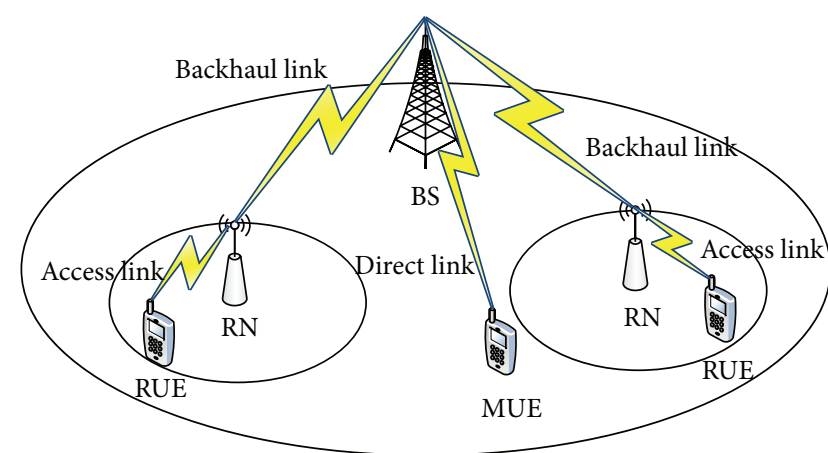

FIGURE 1: The architecture of the LTE-A relay network.

In this paper, we propose a novel energy-saving resource and power allocation scheme in LTE-A relay networks. Our contributions can be summarized as follows. Firstly, to the best of our knowledge, this is the first work to study the energy-conserved uplink resource allocation problem in LTE-A relay networks. The proposed method schedules and allocates radio resource, uplink path, MCS, and transmit power at the same time. Multiple realistic factors are considered in the paper, such as users' required data rate and BER, LTE-A relay network frame structure and system capacity, and the maximum transmit power constraint. Secondly, we prove the problem to be NP-complete. This means that it is impossible to conduct the optimal solution for the problem in limited time. Thirdly, a theoretical analysis is done to show that the complexity of the proposed heuristics is $O\left(n^{2}\right)$. Finally, we conduct a series of simulations to evaluate the performance of the proposed scheme. The simulation results confirm our motivation and show that the proposed method can significantly reduce the overall energy consumption of UE items compared to other schemes, guarantee users' throughput, and increase only few extra delays (less than $10 \mathrm{~ms}$ ).

The rest of the paper is organized as follows. Section 2 gives the preliminaries. Section 3 presents our energyconserved uplink resource allocation heuristics. Theoretical complexity analysis is given in Section 4. Simulation results are shown in Section 5. Section 6 concludes the paper.

\section{Preliminaries}

In this section, we first illustrate and define the system model of LTE-A relay networks. Then, the energy cost model used in this paper is described. Finally, we define the energyconserved uplink resource allocation problem in LTE-A relay networks and prove it to be NP-complete [19]. To facilitate the readability, Notations shown at the end of the paper summarizes the notations frequently used throughout the paper.

2.1. System Model. In an LTE-A relay network, there is one eNB with $M$ fixed relay nodes (RNs) and $N$ UE items, as shown in Figure 1. RNs are deployed to help relay data between cell-edge UE items and eNB to improve the signal 


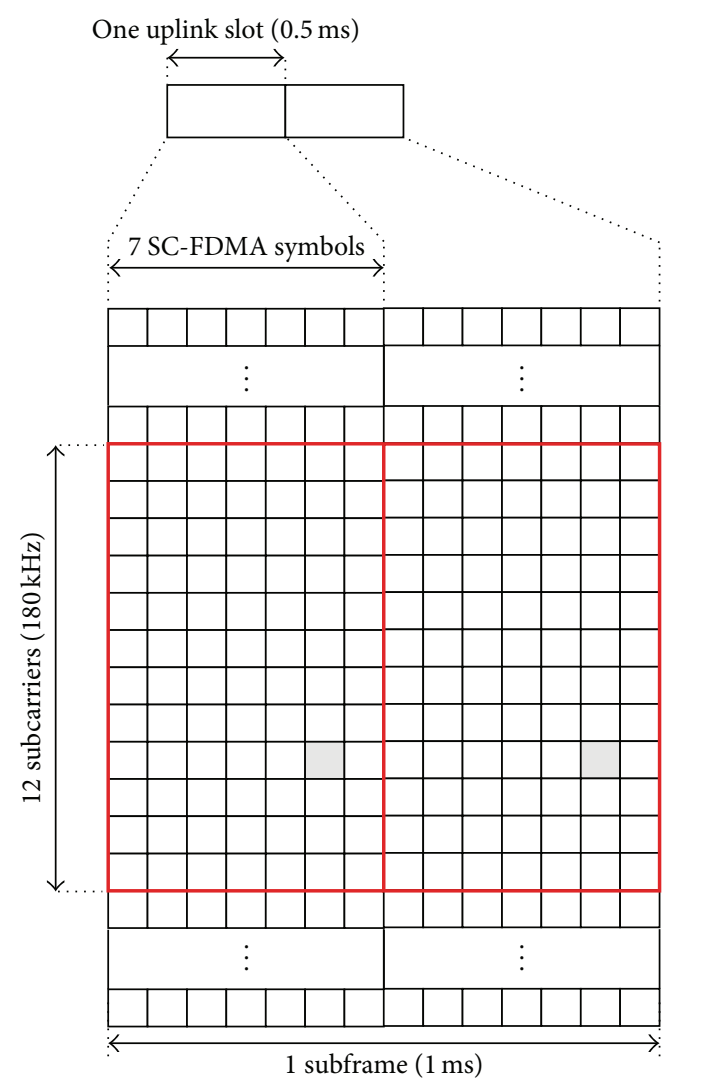

FIgURe 2: One TTI is composed of 2 consecutive RBs, where each $\mathrm{RB}$ is a 12 (subcarriers) $\times 7$ (symbols) two-dimensional array.

quality. There is no direct communication between UE items or RNs. All UE items roam in the eNB's coverage. We call the UE items transmitting data by eNB "MUE" and the UE items transmitting data by RN "RUE." Backhaul links, access links, and direct links are the links between the eNB and RNs, RUEs and RNs, and the eNB and MUEs, respectively. In the relay network, the resource allocation unit is 2 consecutive Resource Blocks (RBs) in time domain, called one Transmission Time Interval (TTI). One RB is a twodimensional array (12 subcarriers $\times 7$ symbols). One TTI with two consecutive RBs is as shown in Figure 2. There are two types of radio frame structures: Time Division Duplex (TDD) mode and Frequency Division Duplex (FDD) mode [20]. In TDD, the radio resource is divided into frames; each is of $10 \mathrm{~ms}$. One frame is composed of 10 subframes of $1 \mathrm{~ms}$ each (as shown in Figure 3), and each subframe is divided into two slots. The LTE-A allows the resource management to schedule the resource on a subframe basis. In other words, the shortest scheduling period in LTE-A is $1 \mathrm{~ms}$. LTEA supports seven different uplink-downlink configurations for the TDD mode as shown in Table 1. Table 2 [21] shows the subframe configurations for eNB-RN (backhaul link) uplink and downlink in LTE-A relay networks. We call the subframes configured for eNB-RN communication "backhaul subframes," in which both the eNB-RN and eNB-MUE communications are allowed. On the contrary, the subframes which are left blank are called "nonbackhaul subframes," in
TABLE 1: TDD frame uplink-downlink configuration.

\begin{tabular}{lcccccccccc}
\hline Uplink-downlink & \multicolumn{1}{c}{ Subframe number } \\
configuration & 0 & 1 & 2 & 3 & 4 & 5 & 6 & 7 & 8 & 9 \\
\hline 0 & D & S & U & U & U & D & S & U & U & U \\
1 & D & S & U & U & D & D & S & U & U & D \\
2 & D & S & U & D & D & D & S & U & D & D \\
3 & D & S & U & U & U & D & D & D & D & D \\
4 & D & S & U & U & D & D & D & D & D & D \\
5 & D & S & U & D & D & D & D & D & D & D \\
6 & D & S & U & U & U & D & S & U & U & D \\
\hline
\end{tabular}

which the RN-RUE and eNB-MUE communications are allowed. Note that in this paper, we skip the FDD mode and focus on the TDD mode. Actually, our method can apply on both LTE-A TDD and FDD modes.

Figure 4 shows an example which demonstrates how subframes are configured in LTE-A relay networks when TDD eNB-RN transmission subframe configuration 1 in Table 2 is used. Since configuration 1 adopts uplink-downlink configuration 1 in Table 1, subframes 2, 3, 7, and 8 are for the uplink and subframes $0,4,5$, and 9 are for the downlink. In the above 8 subframes, subframes 3 and 9 are for uplink and downlink backhaul subframes, respectively, in configuration 1. So, in relay networks, the other 6 subframes, that is, subframes $0,2,4,5,7$, and 8 , are nonbackhaul subframes. In relay networks, the RN-RUE transmission (access link) is only allowed to use the nonbackhaul subframes, while the eNB-RN transmission (backhaul link) can only allocate the resource in the backhaul subframes. The eNB-MUE transmission (direct link) is able to use both kinds of subframes.

2.2. Energy Model. The energy cost of each $\mathrm{UE}_{i}, i=1, \ldots, N$, is $E_{i}=P_{i} \times T_{i}$, where $P_{i}$ is the transmit power (in $\mathrm{mW}$ ) of $\mathrm{UE}_{i}$ and $T_{i}$ is the amount of allocated resources (in TTI or symbol time) to $\mathrm{UE}_{i}$. In each schedule, the required physical resource of $\mathrm{UE}_{i}$ depends on its MCS, MCS ${ }_{i}$, and the data request, $\delta_{i}$ (in bits). $T_{i}$ can be derived by $T_{i}=\left\lceil\delta_{i} / \operatorname{rate}\left(\mathrm{MCS}_{i}\right)\right\rceil$. In fact, LTE-A uses Channel Quality Indicators (CQIs) to report the current channel condition and each CQI $=k, k=1, \ldots, 15$, has its corresponding MCS (denoted by MCS(CQI $=k)$ ) and rate (denoted by rate(CQI $=k$ ); the unit is bits/TTI) [22]. Furthermore, for different CQI and different BER $(\xi)$, it requires different Signal-to-Interference-plus-Noise Ratio (SINR). Figure 5 shows the required SINR over different $\xi$ for different CQIs [23]. With Figure 5, we can get each $\mathrm{UE}_{i}$ 's required SINR, SINR $\left(\mathrm{CQI}_{i}, \xi_{i}\right)$, accordingly. For the communication pair $(i, j)$, the perceived SINR (in $\mathrm{dB}$ ) of receiver $j$ can be written as

$$
\operatorname{SINR}_{i, j}=10 \times \log _{10} \frac{P_{i, j}}{B \times N_{0}+I_{i, j}},
$$

where $P_{i, j}$ is the received power at receiver $j, B$ is the effective bandwidth (in $\mathrm{Hz}$ ), $N_{0}$ is the thermal noise level, and $I_{i, j}$ is the interference from transmitters other than $i$, which can 


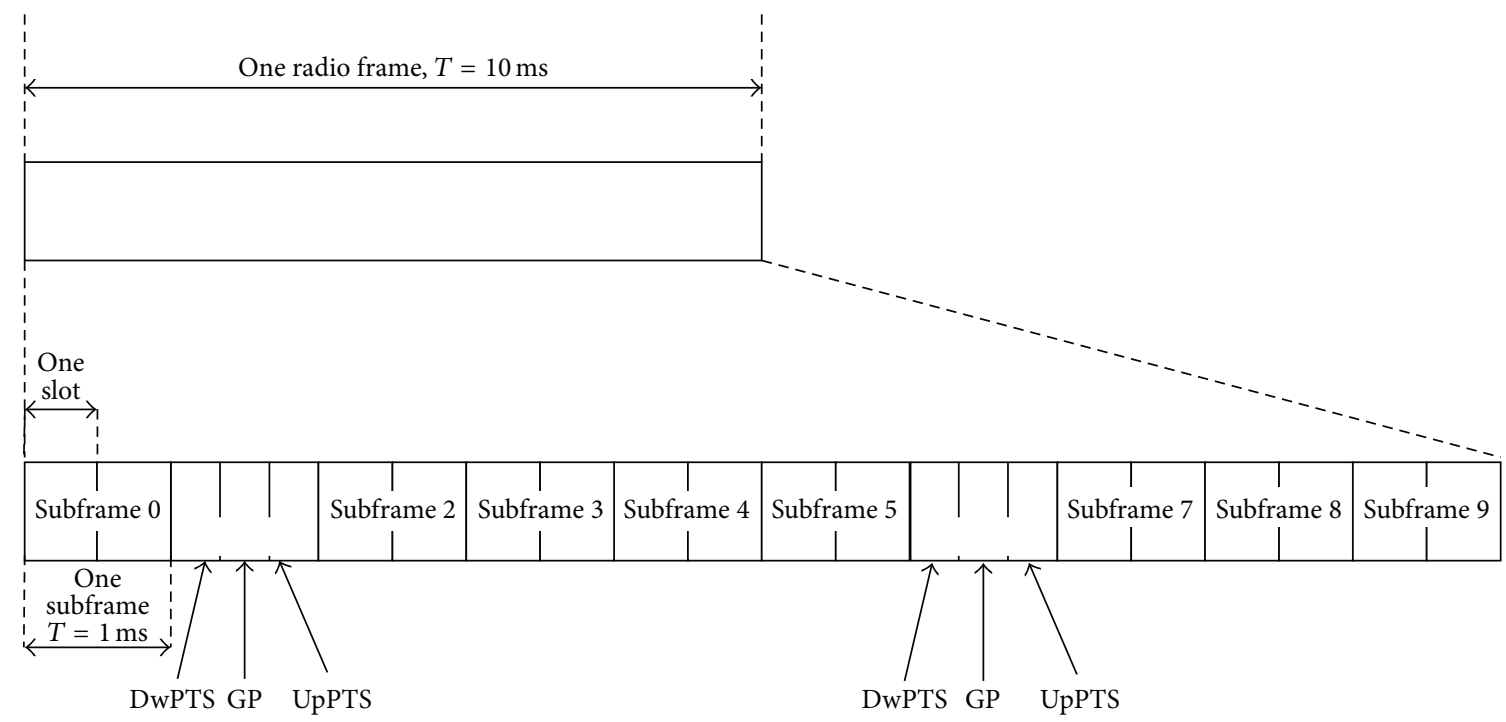

Figure 3: Frame structure of LTE-A TDD mode.

\begin{tabular}{|c|c|c|c|c|c|c|c|c|c|c|}
\hline $\begin{array}{c}\text { Subframe } \\
\text { number }\end{array}$ & 0 & 1 & 2 & 3 & 4 & 5 & 6 & 7 & 8 & 9 \\
\hline $\begin{array}{c}\text { Subframe } \\
\text { configuration }\end{array}$ & $\mathrm{nB}-\mathrm{D}$ & $\mathrm{S}$ & $\mathrm{nB}-\mathrm{U}$ & $\mathrm{B}-\mathrm{U}$ & $\mathrm{nB}-\mathrm{D}$ & $\mathrm{nB}-\mathrm{D}$ & $\mathrm{S}$ & $\mathrm{nB}-\mathrm{U}$ & $\mathrm{nB}-\mathrm{U}$ & $\mathrm{B}-\mathrm{D}$ \\
\hline
\end{tabular}

nB-U/D: nonbackhaul uplink/downlink subframe

B-U/D: backhaul uplink/downlink subframe

FIGURE 4: A subframe configuration example for LTE-A relay networks with TDD eNB-RN transmission subframe configuration 1.

TABLE 2: Supported configurations for TDD eNB-RN transmission.

\begin{tabular}{|c|c|c|c|c|c|c|c|c|c|c|c|}
\hline \multirow{2}{*}{ Subframe configuration } & \multirow{2}{*}{$\begin{array}{l}\text { eNB-RN uplink-downlink } \\
\text { configuration }\end{array}$} & \multicolumn{10}{|c|}{ Subframe number } \\
\hline & & 0 & 1 & 2 & 3 & 4 & 5 & 6 & 7 & 8 & 9 \\
\hline 0 & & & & & & $\mathrm{D}$ & & & & $\mathrm{U}$ & \\
\hline 1 & & & & & $\mathrm{U}$ & & & & & & $\mathrm{D}$ \\
\hline 2 & 1 & & & & & $\mathrm{D}$ & & & & $\mathrm{U}$ & $\mathrm{D}$ \\
\hline 3 & & & & & $\mathrm{U}$ & $\mathrm{D}$ & & & & & $\mathrm{D}$ \\
\hline 4 & & & & & $\mathrm{U}$ & $\mathrm{D}$ & & & & $\mathrm{U}$ & $\mathrm{D}$ \\
\hline 5 & & & & $\mathrm{U}$ & & & & & & $\mathrm{D}$ & \\
\hline 6 & & & & & $\mathrm{D}$ & & & & $\mathrm{U}$ & & \\
\hline 7 & 2 & & & $\mathrm{U}$ & & $\mathrm{D}$ & & & & $\mathrm{D}$ & \\
\hline 8 & & & & & $\mathrm{D}$ & & & & $\mathrm{U}$ & & $\mathrm{D}$ \\
\hline 9 & & & & $\mathrm{U}$ & $\mathrm{D}$ & $\mathrm{D}$ & & & & $\mathrm{D}$ & \\
\hline 10 & & & & & $\mathrm{D}$ & & & & $\mathrm{U}$ & $\mathrm{D}$ & $\mathrm{D}$ \\
\hline 11 & 3 & & & & $\mathrm{U}$ & & & & $\mathrm{D}$ & & $\mathrm{D}$ \\
\hline 12 & 3 & & & & $\mathrm{U}$ & & & & $\mathrm{D}$ & $\mathrm{D}$ & $\mathrm{D}$ \\
\hline 13 & & & & & $\mathrm{U}$ & & & & & & $\mathrm{D}$ \\
\hline 14 & & & & & $\mathrm{U}$ & & & & $\mathrm{D}$ & & $\mathrm{D}$ \\
\hline 15 & 4 & & & & $\mathrm{U}$ & & & & & $\mathrm{D}$ & $\mathrm{D}$ \\
\hline 16 & & & & & $\mathrm{U}$ & & & & $\mathrm{D}$ & $\mathrm{D}$ & $\mathrm{D}$ \\
\hline 17 & & & & & $\mathrm{U}$ & $\mathrm{D}$ & & & $\mathrm{D}$ & $\mathrm{D}$ & $\mathrm{D}$ \\
\hline 18 & 5 & & & & & $\mathrm{U}$ & & & & & $\mathrm{D}$ \\
\hline
\end{tabular}




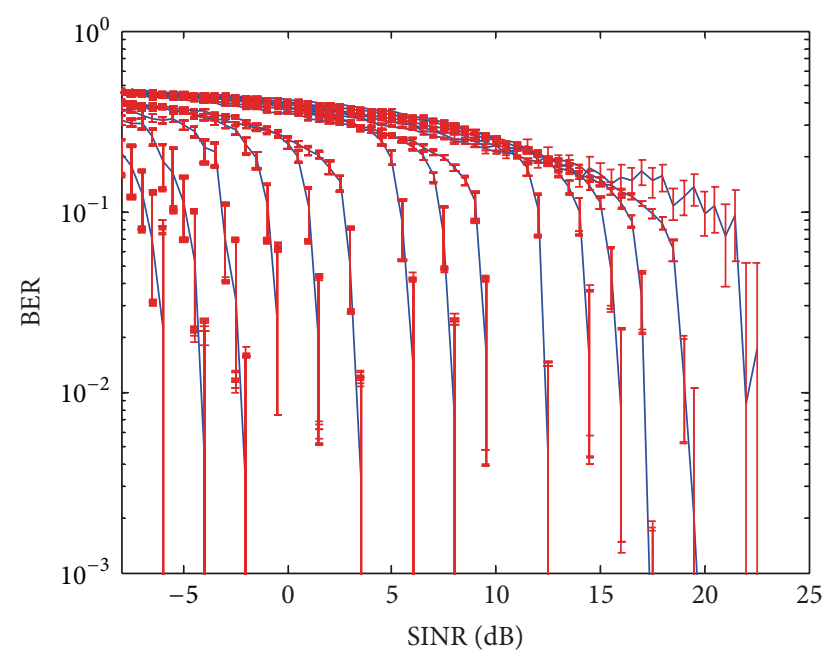

FIGURE 5: Error ratio for different CQIs (the 99\% confidence intervals are depicted in red).

be evaluated by $I_{i, j}=\sum_{i \neq j} P_{i, j}$. Ignoring shadow and fading effect, $P_{i, j}$ can be derived by

$$
P_{i, j}=\frac{G_{i} \times G_{j} \times P_{i}}{L_{i, j}}
$$

where $G_{i}$ and $G_{j}$ are the antenna gains at $\mathrm{UE}_{i}$ and $\mathrm{RN}_{j}$, respectively, and $L_{i, j}$ is the path loss from $i\left(\mathrm{UE}_{i}\right)$ to $j\left(\mathrm{RN}_{j}\right.$ or the eNB). To save $\mathrm{UE}_{i}$ 's energy, we can minimize its transmit power subject to the required minimum SINR; that is, using $\operatorname{MCS}\left(\mathrm{CQI}_{i}=k\right), \mathrm{UE}_{i}$ 's data can be correctly decoded by receiver $j$ with a guaranteed BER $\xi_{i}$ only when

$$
\operatorname{SINR}_{i, j} \geq \operatorname{SINR}\left(\mathrm{CQI}_{i}=k, \xi_{i}\right) .
$$

Consequently, by integrating (1), (2), and (3), the required transmit power $P_{i}$ of $\mathrm{UE}_{i}$ subject to the applied MCS $\left(\mathrm{CQI}_{i}\right)$ and requested $\xi_{i}$ for the communication pair $(i, j)$ is

$$
P_{i} \geq \frac{10^{\operatorname{SINR}\left(\mathrm{CQI}_{i}, \xi_{i}\right) / 10} \times\left(B \times N_{0}+I_{i, j}\right) \times L_{i, j}}{G_{i} \times G_{j}} .
$$

2.3. Problem Definition. The uplink energy conservation problem is defined as below. We assume that, in the LTEA relay network, there is one eNB with $M$ fixed RNs and $N$ UE items. For each $\mathrm{UE}_{i}, i=1, \ldots, N$, it has an average uplink traffic demand $\delta_{i}$ bits/frame granted by the resource management of the eNB. UE items can uplink data to the eNB either directly or indirectly through RNs. Suppose that the relative distances between eNB/RNs and UE items can be estimated through existing techniques. The objective of the problem is to minimize the total energy consumption of UE items, while guaranteeing their required $\xi_{i}$ and traffic demands being all delivered to the eNB subject to the total amount of physical resources and the maximum transmit power constraints. Without loss of generality, we assume that the total amounts of physical resources for backhaul and nonbackhaul subframes are $F_{\mathrm{B}}$ and $F_{\mathrm{nB}}$ TTIs per frame, respectively. To solve the problem, we have to determine the uplink path, resource allocation, uplink transmit power $P_{i}$, and the used $\mathrm{CQI}_{i}$ of each $\mathrm{UE}_{i}$.

Theorem 1. The energy conservation problem is NP-complete.

Proof. To simplify the proof, we consider the case of no spatial reuse in the UE-RN transmissions and each UE has already selected an appropriate $\mathrm{RN}$ according to the channel condition. So, each UE can select an MCS to deliver data to RN and each MCS costs different energy consumption. Thus, the energy cost of one UE item using a specific MCS is uniquely determined. Then, we formulate the uplink resource allocation problem as a decision problem, energy-conserved uplink resource allocation decision (EURAD) problem, as below. Given the network topology $G$ and the demand of each UE item, we ask whether or not there exists one MCS set $S_{\text {MCS }}$ such that, with the corresponding selected MCSs, all UE items can conserve the total amount of energy $Q$ and satisfy each of their demands and the total amount of required RBs is not greater than the frame size $U$. Then, we will show EURAD problem to be NP-complete.

We first show that the EURAD problem belongs to NP. Given a problem instance and a solution containing the MCS set, it definitely can be verified whether or not the solution is valid in polynomial time. Thus, this part is proved.

We then reduce the multiple-choice knapsack (MCK) problem [24], which is known to be NP-complete, to the EURAD problem. When the reduction is done, the EURAD problem is proved to be NP-complete.

Before the reduction, let us briefly introduce the MCK problem first. The MCK problem is a problem in combinatorial optimization: Given a set of $n$ disjointed classes of objects, where each class $i$ contains $N_{i}$ objects, for each object $X_{i, j}, i=1, \ldots, n, j=1, \ldots, N_{i}$, it has a weight $u_{i, j}$ and a profit $q_{i, j}$. For each class $i$, one and only one object must be selected, that is, $\sum_{\forall j=1}^{N_{i}} I_{i, j}=1, i=1, \ldots, n$, where $I_{i, j}=1$ when object $X_{i, j}$ is picked and chosen; otherwise, $I_{i, j}=0$. The problem is to determine which $n$ objects shall be included in a knapsack to maximize the total object profit and the total weight has to be less than or equal to a given $\operatorname{limit} U$ and $U$ is also called the capacity constraint. So, the MCK problem can be formally formulated as below:

$$
\max \sum_{\forall i=1 \forall j=1}^{n} \sum_{i, j}^{N_{i}} q_{i, j} I_{i, j}
$$

subject to $\sum_{\forall i=1 \forall j=1}^{n} \sum_{i, j}^{N_{i}} u_{i, j} I_{i, j} \leq U$,

$$
\begin{aligned}
& \sum_{\forall j=1}^{N_{i}} I_{i, j}=1, \quad i=1, \ldots, n, \\
& I_{i, j}=\{0,1\}, \quad i=1, \ldots, n, j=1, \ldots, N_{i} .
\end{aligned}
$$

To reduce the MCK problem to the EURAD problem, an instance of the MCK problem is constructed as below. 
Consider that there are $n$ disjointed classes of objects, where each class $i$ contains $N_{i}$ objects. In each class $i$, every object $X_{i, j}$ has a profit $q_{i, j}$ and a weight $u_{i, j}$. Besides, there is a knapsack with capacity of $U$. The MCK problem is no larger than $U$ and the total object profit is $Q$.

An instance of the EURAD problem is also constructed as follows. Let $n$ be the number of UE items. Each $\mathrm{UE}_{i}$ has $N_{i}$ MCSs to its connected eNB/RN. When $\mathrm{UE}_{i}$ selects one $\operatorname{MCS} x_{i, j}, j=1, \ldots, N_{i}$, it will conserve energy of $q_{i, j}$ (which is compared to the energy consumption when $\mathrm{UE}_{i}$ uses its best level of MCS) and the system should allocate RB(s) of a total size of $u_{i, j}$ to transmit $\mathrm{UE}_{i}$ 's data to the connected $\mathrm{eNB} / \mathrm{RN}$. The total frame space is $U$. Our goal is to let all UE items conserve energy of $Q$ and satisfy their demands. In the following, we will show that the MCK problem has a solution if and only if the EURAD problem has a solution.

Suppose that we have a solution to the EURAD problem, which is one MCS set $S_{\text {MCS }}$ with UE items' conserved energy and RB allocations. Each UE item chooses exact one MCS which is able to satisfy its demand. The total size of required RBs cannot exceed $U$ and the conserved energy of all UE items is $Q$. By viewing the available MCSs of one UE item as a class of objects and the total number of RBs $U$ as the capacity of the knapsack, the MCSs in $S_{\text {MCS }}$ constitute a solution to the MCK problem. This proves the only if part.

Conversely, let $\left\{x_{1, \alpha_{1}}, x_{2, \alpha_{2}}, \ldots, x_{n, \alpha_{n}}\right\}$ be a solution to the MCK problem. Then, for each $\mathrm{UE}_{i}, i=1, \ldots, n$, we select one MCS such that $\mathrm{UE}_{i}$ conserves energy of $q_{i, \alpha_{i}}$ and the number of allocated $\mathrm{RB}(\mathrm{s})$ to transmit $\mathrm{UE}_{i}$ 's data to its connected $\mathrm{eNB} / \mathrm{RN}$ is $u_{i, \alpha_{i}}$. In this way, the conserved energy of all UE items will be $Q$ and the overall $R B$ is no larger than $U$. This constitutes a solution to the EURAD problem, thus proving the only if part.

\section{Proposed Method}

This section illustrates our proposed heuristics. The method is composed of two phases. In the first phase, each UE selects an uplink path according to the channel condition and adopts the lowest level of MCS, that is, MCS(CQI = 1), for power saving. If the amount of required radio resources of UE items exceeds the system capacity, the second phase is then executed. The second phase exploits spatial reuse (or concurrent transmission) and high level of MCS to increase the radio resource usage efficiency. LTE-A relay networks allow multiple UE items to utilize the same radio resource and transmit concurrently to each of their serving RNs in nonbackhaul subframes, called spatial reuse. Both spatial reuse and high level MCSs help the reduction of total required TTIs of the system. In the end, the total amounts of required TTIs must meet the system capacity $F_{\mathrm{B}}$ and $F_{\mathrm{nB}}$, and UE items' requirements have to be guaranteed.

3.1. Phase I: Initialization and Uplink Path Selection. There are $M+1$ candidate uplink paths for UE items, that is, $\mathrm{RN}_{j}$, $j=0, \ldots, M$. Note that $\mathrm{RN}_{0}$ is used to represent the central eNB. Initially, set $S_{j}^{R}=\emptyset$ for each $\mathrm{RN}_{j}$. Then, for each $\mathrm{UE}_{i}, i=$ $1, \ldots, N$, select the $\mathrm{RN}_{j^{*}}$, where $j^{*}=\arg \max _{\forall j}\left\{\operatorname{SINR}_{i, j}\right\}$, as the uplink path and set $S_{j^{*}}^{R}=S_{j^{*}}^{R}+\mathrm{UE}_{i}$. To minimize $E_{\text {total }}$, each $\mathrm{UE}_{i}$ applies $\mathrm{CQI}_{i}=1$. This leads to eNB/RNs must allocate more RBs to UE items. But, in phase I, we omit the total radio resource constraint temporarily. The required amount of TTIs for $\mathrm{UE}_{i}$ to deliver data to its connecting $\mathrm{RN}_{j}$ can be derived by

$$
T_{i}^{\mathrm{UE} \_\mathrm{RN}}=\left\lceil\frac{\delta_{i}}{\operatorname{rate}\left(\mathrm{CQI}_{i}=1\right)}\right\rceil ;
$$

subsequently, $\mathrm{RN}_{j}$ requires radio resource $T_{i}^{\mathrm{RN} \_\mathrm{BS}}$ in backhaul subframes to forward the received data to the eNB. $T_{i}^{\mathrm{RN} \_\mathrm{BS}}$ can be conducted by

$$
T_{i}^{\mathrm{RN} \_ \text {BS }}=\sum_{j=1, \ldots, M} x_{i, j} \times\left\lceil\frac{\delta_{i}}{\operatorname{rate}(\mathrm{CQI}=15)}\right\rceil,
$$

where $x_{i, j}=1$ when $\mathrm{RN}_{j}$ is $\mathrm{UE}_{i}$ 's uplink path; otherwise, $x_{i, j}=0$. Then check whether $\sum_{\forall i, x_{i, 0} \neq 1} T_{i}^{\mathrm{UE} \_\mathrm{RN}} \leq F_{\mathrm{nB}}$ and $\sum_{i=1}^{N}\left(T_{i}^{\mathrm{RN} \_\mathrm{BS}}+T_{i}^{\mathrm{UE} \_\mathrm{RN}}\right) \leq F_{\mathrm{B}}+F_{\mathrm{nB}}$ or not. If yes, terminate the algorithm and return each $\mathrm{UE}_{i}$ 's resource allocation $\left(T_{i}^{\mathrm{UE} \_\mathrm{RN}}\right.$ and $\left.T_{i}^{\mathrm{RN} \_\mathrm{BS}}\right)$, uplink path, MCS, and uplink transmit power $P_{i}=\left(10^{\operatorname{SINR}\left(\mathrm{CQI}_{i}, \xi_{i}\right) / 10} \times B \times N_{0} \times L_{i, j}\right) /\left(G_{i} \times G_{j}\right)($ refer to $(4))$. Otherwise, go to phase II for further execution.

3.2. Phase II: Energy-Saving Resource Allocation. Phase II is to satisfy UE items' requests with the least additional energy consumption. To reduce the total amount of required RBs, we first exploit the concurrent transmission. In a concurrent transmission group, $g_{k}$, member UE items connect to different eNB/RNs and use the same RBs to deliver data. This reduces the demand of UE items in $g_{k}$ from $\sum_{\forall i \in g_{k}} T_{i}^{\mathrm{UE} \_\mathrm{RN}}$ to $\max \left\{T_{i}^{\mathrm{UE} \_\mathrm{RN}} \mid \forall i \in g_{k}\right\}$. However, the UE items in the same group will interfere with each other such that the UE items have to spend extra transmit power to guarantee $\xi_{i}$. To minimize the additional power consumption, we have to find interference-free UE items to form groups. Hence, a weight function $\left(W_{i}\right)$ is defined to evaluate UE items in the network. $W_{i}$ of $\mathrm{UE}_{i}, i=1, \ldots, N$, can be expressed by

$$
\begin{aligned}
& W_{i} \\
& =\alpha \times \frac{\left(d_{i, j}\right)^{-w}}{\left(\min _{\ell=1, \ldots, N}\left\{d_{\ell, j} \mid x_{\ell, j} \neq 0\right\}\right)^{-w}}+\beta \\
& \quad \times \frac{\delta_{i}}{\max _{\ell=1, \ldots, N}\left\{\delta_{\ell} \mid x_{\ell, j} \neq 0\right\}}+(-\gamma) \\
& \quad \times\left(1+\Delta \times t_{i}\right) \\
& \quad \times \sum_{\forall v, v \neq j,\left(\sum_{\ell=1}^{N} x_{\ell, v}\right) \neq 0} \frac{\left(d_{i, v}\right)^{-w}}{\left(\min _{\ell=1, \ldots, N}\left\{d_{\ell, v} \mid x_{\ell, v} \neq 0\right\}\right)^{-w}},
\end{aligned}
$$

where $\alpha, \beta$, and $\gamma$ are normalized coefficients and $\alpha+\beta-\gamma=1$, $w$ is the spreading factor, $t_{i}$ denotes the number of times that $\mathrm{UE}_{i}$ has been excluded from concurrent transmission 
groups, and $\Delta$ is the normalized coefficient. The values of the three coefficients, $\alpha, \beta$, and $\gamma$, control the relative importance of three factors, path loss, data quantity, and interference, respectively. To form $g_{k}$, for each $\mathrm{RN}_{j}, j=0, \ldots, M$, we choose one ungrouped UE item with the maximum weight in all UE items connecting to $\mathrm{RN}_{j}$, that is, $i^{*}=\arg \max _{\forall i \in S_{j}^{R}}\left\{W_{i}\right\}$. Then, calculate the required transmission power $\widehat{P}_{i}$ of each $\mathrm{UE}_{i}$ in $g_{k}$, where $\widehat{P}_{i}$ must be able to guarantee $\xi_{i}$. To prevent $g_{k}$ from selecting the UE items which seriously interfere with others or are interfered with, we will check whether $E_{k}=$ $\sum_{\forall i, i \in g_{k}}\left(\widehat{P}_{i} \times T_{i}^{\mathrm{UE} \_\mathrm{RN}}\right)$ is greater than the energy threshold $E_{k}^{\text {th }}$ or not. If yes, it means that some communication pairs suffer great interference from other UE items in $g_{k}$. The threshold $E_{k}^{\text {th }}$ is set to the summation of the required transmit energy of all UE items in $g_{k}$ as concurrent transmission is not applied and the same amount of TTIs is consumed as the case of concurrent transmission. If serious interference exists in $g_{k}$, the exclusion algorithm will be triggered to remove some UE items from $g_{k}$. The detail of the exclusion algorithm will be described later. After all UE items are assigned concurrent transmission groups, if UE items' requests are still not satisfied, we consider increasing the MCS level of UE items.

For each $g_{k}, k=1, \ldots, K$ (assume there are totally $K$ concurrent transmission groups and $K \leq N$ ), we first calculate the energy consumption and required number of $\mathrm{RBs}$ of all feasible CQI settings. We define the penalty function $P_{f}(k, x, y)$ to evaluate $g_{k}$ 's penalty when changing its CQI setting from a low level $x$ to a high level $y$, where $x$ and $y$ are vectors. The penalty function is defined as

$$
P_{f}(k, x, y)=\frac{\Delta E_{x, y}^{k}}{\Delta A_{x, y}^{k}}=\frac{E_{y}^{k}-E_{x}^{k}}{A_{x}^{k}-A_{y}^{k}},
$$

where $E_{y}^{k}$ and $E_{x}^{k}$ are the amount of energy consumption of $g_{k}$ using $\operatorname{MCS}\left(\mathrm{CQI}_{g_{k}}=y\right)$ and $\operatorname{MCS}\left(\mathrm{CQI}_{g_{k}}=x\right)$, respectively, and $A_{x}^{k}$ and $A_{y}^{k}$ are the number of required $\mathrm{RBs}$ of $g_{k}$ by adopting $\operatorname{MCS}\left(\mathrm{CQI}_{g_{k}}=x\right)$ and $\operatorname{MCS}\left(\mathrm{CQI}_{g_{k}}=y\right)$, respectively. The group with the least penalty is preferred to upgrade its CQIs. Note that uplink resource arrangement has to follow the resource constraints of backhaul and nonbackhaul subframes. The algorithm of phase II is as below.

(1) For each $\mathrm{UE}_{i}, i=1, \ldots, N$, calculate $W_{i}$.

(2) Set $S_{j}^{R^{\prime}}=S_{j}^{R}$ for $j=0, \ldots, M, S=\left\{\mathrm{UE}_{i}, i=\right.$ $1, \ldots, N\}, k=1, T_{\text {all }}^{\text {access }}=\sum_{\forall i, x_{i, 0} \neq 1} T_{i}^{\mathrm{UE} \_\mathrm{RN}}$, and $T_{\mathrm{all}}=\sum_{i=1}^{N}\left(T_{i}^{\mathrm{RN} \_\mathrm{BS}}+T_{i}^{\mathrm{UE} \_\mathrm{RN}}\right)$.

(3) For each $S_{j}^{R^{\prime}}$, choose the $\mathrm{UE}_{i^{*}} \in S_{j}^{R^{\prime}}$, where $i^{*}=$ $\arg \max _{\forall \mathrm{UE}_{i} \in S_{j}^{R^{\prime}}}\left\{W_{i}\right\}$, and set $g_{k}=g_{k}+\mathrm{UE}_{i^{*}}$.

(4) Calculate $\widehat{P}_{i}$ for each $\mathrm{UE}_{i} \in g_{k}$ (refer to (4)). If $E_{k} \leq E_{k}^{\text {th }}$, go to the next step; otherwise, execute the exclusion algorithm to remove the most infeasible UE from $g_{k}$ (assume it is $\mathrm{UE}_{\ell}$ ). Then set $g_{k}=g_{k}-\left\{\mathrm{UE}_{\ell}\right\}$ and update $t_{\ell}=t_{\ell}+1$ and $W_{\ell}$. Repeat step (4).
(5) If $\left|g_{k}\right|>1$, update $T_{\text {all }}^{\text {access }}=T_{\text {all }}^{\text {access }}-$ $\sum_{\forall i \in g_{k}, x_{i, 0} \neq 1} T_{i}^{\mathrm{UE} \_\mathrm{RN}}+\max \left\{T_{i}^{\mathrm{UE} \_\mathrm{RN}} \mid \forall i \in g_{k}\right\}$ and $T_{\text {all }}=T_{\text {all }}-\sum_{\forall i \in g_{k}} T_{i}^{\mathrm{UE} \_ \text {RN }}+\max \left\{T_{i}^{\mathrm{UE} \_\mathrm{RN}} \mid \forall i \in g_{k}\right\}$. Set $S_{j}^{R^{\prime}}=S_{j}^{R^{\prime}}-g_{k}$ for $j=0, \ldots, M$ and $S=S-g_{k}$. If $T_{\text {all }}^{\text {access }} \leq F_{\mathrm{nB}}$ and $T_{\mathrm{all}} \leq F_{\mathrm{B}}+F_{\mathrm{nB}}$, terminate the algorithm and return the result of resource allocation, grouping, uplink path, MCS configuration, and uplink transmit power. If $S \neq \emptyset$, go back to step (3); otherwise, go to the next step.

(6) For each group $g_{k}, k=1, \ldots, K$, form the MCS configuration pattern matrix $A_{k}=\left[x_{1}^{k}, \ldots, x_{\mathfrak{\Im}_{k}}^{k}\right]$, where $x_{\wp}^{k}=\left[x_{\wp, 1}^{k}, \ldots, x_{\wp,\left|g_{k}\right|}^{k}\right]^{T}$ and $x_{\wp}^{k}$ is one of feasible MCS configuration patterns for $g_{k}$. Then, calculate the energy consumption $E_{\wp}^{k}$ and the number of required RBs $T_{\wp}^{\mathrm{UE} \_\mathrm{RN}, k}$ for each $x_{\wp}^{k}$. Note that, without loss of generality, we assume that $E_{1}^{k} \leq \cdots \leq E_{\mathfrak{\Im}_{k}}^{k}$ and $T_{1}^{\mathrm{UE} \_ \text {RN,k}} \geq \cdots \geq T_{\mathfrak{\Im}_{k}}^{\mathrm{UE} \_\mathrm{RN}, k}$ (how to efficiently form the $\mathfrak{\Im}_{k}$ feasible MCS configuration patterns for $g_{k}$ is discussed in Section 3.4).

(7) For each $g_{k}$, calculate the penalties from $x_{1}^{k}$ to all possible MCS configuration $x_{\wp}^{k}, \wp=2, \ldots, \mathfrak{\Im}_{k}$.

(8) First, consider the set of groups $A$ which can only be assigned resource in $F_{\mathrm{nB}}$, that is, $A=\left\{g_{k} \mid\right.$ $\left.\exists i \in g_{k}: x_{i, 0}=0\right\}$. For all groups in $A$, select the minimum $P_{f}\left(k^{*}, x^{*}, y^{*}\right)$ and then change $g_{k^{*}}$ 's MCS configuration from $x^{*}$ to $y^{*}$, update $g_{k}$ 's required physical resource and transmit power, and recalculate its penalties from $y^{*}$ to $x_{\wp}^{k}, \wp=\left(y^{*}+1\right), \ldots, \Im_{k}$. Check whether new $T_{\text {all }}^{\text {access }} \leq F_{\mathrm{nB}}$ or not. If yes, go to the next step; otherwise, repeat step (8).

(9) In this step, we consider satisfying the $F_{\mathrm{B}}+F_{\mathrm{nB}}$ constraint. The operation is the same as the previous step but we set $A=\left\{g_{k} \mid \forall k\right\}$. Each time after changing a group's MCS configuration (assume it is group $g_{k^{*}}$, , check whether new $T_{\text {all }} \leq F_{\mathrm{B}}+F_{\mathrm{nB}}$ or not. If yes, stop the algorithm and return each $\mathrm{UE}_{i}$ 's, $i=1, \ldots, N$, resource allocation, grouping result, uplink path, MCS, and transmit power; otherwise, repeat step (9).

3.3. Exclusion Algorithm. When $E_{k}>E_{k}^{\text {th }}$, it represents that some UE items in $g_{k}$ cause severe interference with other concurrent transmission pairs in the group. We use Figure 6 to explain this. Assume that $\mathrm{UE}_{0}, \mathrm{UE}_{1}, \mathrm{UE}_{2}$, and $\mathrm{UE}_{3}$ are in a concurrent transmission group and $\mathrm{RN}_{0}$ (i.e., eNB), $\mathrm{RN}_{1}$, $\mathrm{RN}_{2}$, and $\mathrm{RN}_{3}$ are their serving base stations, respectively. Take $\mathrm{UE}_{1}$ and its serving base station $\mathrm{RN}_{1}$, for example; Figures 6(a) and 6(b) show the received interference and transmit interference, respectively. As shown in Figure 6(a), for $\mathrm{UE}_{1}$ and $\mathrm{RN}_{1}$, the received interference $I_{1,1}^{r}=P_{0,1}+P_{2,1}+$ $P_{3,1}$. On the other hand, the transmit interference generated by the transmission pair $\left(\mathrm{UE}_{1}, \mathrm{RN}_{1}\right)$ can be calculated by $I_{1,1}^{t}=P_{1,0}+P_{1,2}+P_{1,3}$. Sum up $I_{1,1}^{r}$ and $I_{1,1}^{t}$; we then derive the total interference $I_{1,1}^{\text {sum }}$ of the transmission pair $\left(\mathrm{UE}_{1}, \mathrm{RN}_{1}\right)$. 


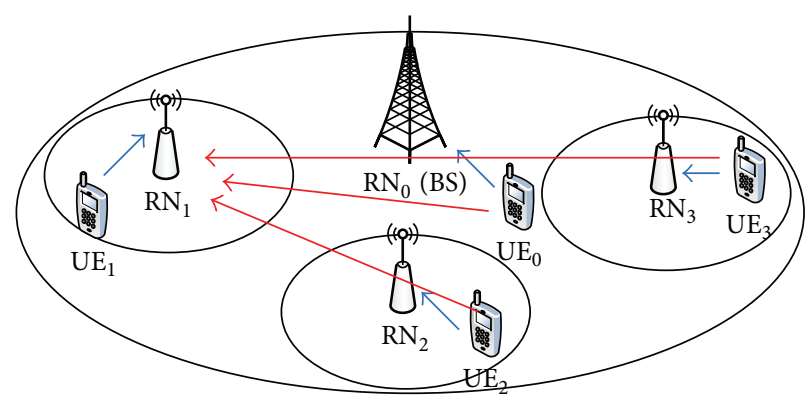

(a) Received interference for $\left(\mathrm{UE}_{1}, \mathrm{RN}_{1}\right)$

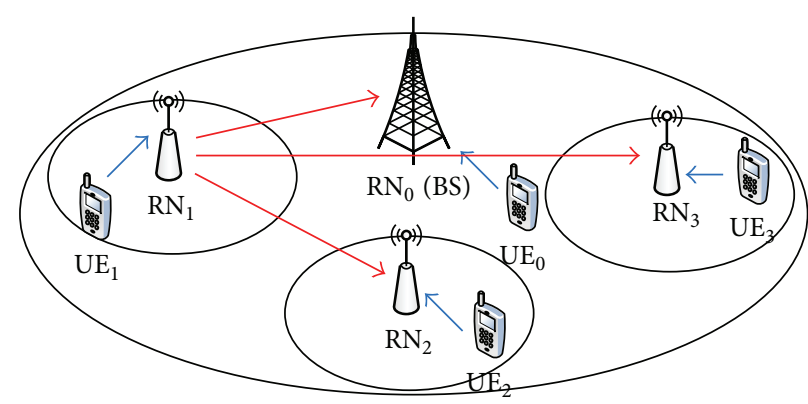

(b) Transmit interference from $\mathrm{UE}_{1}$

FIgURE 6: An example of the total interference of a transmission pair $\left(\mathrm{UE}_{1}, \mathrm{RN}_{1}\right)$.

When $E_{k}>E_{k}^{\text {th }}$ occurs, we must exclude the UE, which causes severe interference, from $g_{k}$ to increase the energy efficiency. The detail is as follows:

(1) Without loss of generality, for the UE items in $g_{k}$, we reindex them as $\mathrm{UE}_{m}, m=1, \ldots,\left|g_{k}\right|$, and denote the set of their uplink eNB/RNs by $\epsilon_{k}$. Next, for each $\mathrm{UE}_{m}$ and its corresponding $\mathrm{RN}_{n}$, calculate the received interference $I_{m, n}^{r}$ by

$$
I_{m, n}^{r}=\sum_{\forall \mathrm{UE}_{\alpha} \in g_{k}, \alpha \neq m} P_{\alpha, n} .
$$

Then, for each $\mathrm{UE}_{m}$, calculate the transmit interference $I_{m, n}^{t}$ as follows:

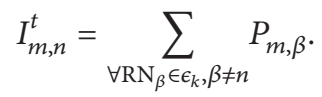

(2) For each $\mathrm{UE}_{m}, m=1, \ldots,\left|g_{k}\right|$, calculate $I_{m, n}^{\mathrm{sum}}=I_{m, n}^{r}+$ $I_{m, n}^{t}$.

(3) From all derived $I_{m, n}^{\text {sum }}$ in the previous step, select the maximum one $I_{m^{*}, n^{*}}^{\text {sum }}$ and exclude the pair $\left(m^{*}, n^{*}\right)$ from $g_{k}$.

3.4. Listing All $\mathfrak{\Im}_{k}$ Feasible MCS Configuration Patterns for $g_{k}$. For each $g_{k}$, the number of possible MCS configurations is $15^{\left|g_{k}\right|}$. Listing and trying all the configurations will have a tremendous cost. Actually, for a group $g_{k}$, only $15 \times\left|g_{k}\right|$ combinations out of $15^{\left|g_{k}\right|}$ (even less) need to be considered. Let us discuss this. Consider a group, $g_{k}=\left\{\mathrm{UE}_{1}, \ldots, \mathrm{UE}_{\left|g_{k}\right|}\right\}$, and one of its MCS configurations, $x_{\wp}^{k}=\left[x_{\wp, 1}^{k}, \ldots, x_{\wp,\left|g_{k}\right|}^{k}\right]^{T}$; assume that applying $x_{\wp}^{k}$ would consume resource $T_{\wp}^{\mathrm{UE} \_\mathrm{RN}, k}=$ $\max \left\{T_{i}^{\mathrm{UE} \_\mathrm{RN}}\left(x_{\wp, i}^{k}\right) \mid \forall i\right\}=T_{1}^{\mathrm{UE} \_\mathrm{RN}}\left(x_{\wp, 1}^{k}\right)$; that is, $\mathrm{UE}_{1}$ requires the largest number of RBs in $g_{k}$ as $x_{\wp}^{k}$ is used. In this case, enhancing any UE's MCS other than $\mathrm{UE}_{1}$ in $g_{k}$ does not reduce the amount of required radio resources but only increases the energy consumption of $g_{k}$. This means that MCS configurations $\left[x_{\wp, 1}^{k},\left(x_{\wp, 2}^{k}+1\right) \cdots 15,\left(x_{\wp, 3}^{k}+\right.\right.$ 1) $\left.\cdots 15, \ldots,\left(x_{\wp,\left|g_{k}\right|}^{k}+1\right) \cdots 15\right]^{T}$ do not have to be taken into account. In other words, each time, only the UE with the largest amount of required RBs has to be considered. In this way, we can greatly reduce the computing complexity. The detailed procedure of listing all feasible MCS configuration patterns for a concurrent transmission group $g_{k}$ is stated as below.

(1) For a group $g_{k}$, initialize all member UE items' MCS level to MCS(CQI = 1). Calculate each of their required amounts of RBs and the total amount of energy consumption. Set $\wp=1$ and $x_{\wp}^{k}=\left[x_{\wp, 1}^{k}=\right.$ $\left.\operatorname{MCS}(\mathrm{CQI}=1), \ldots, x_{\wp,\left|g_{k}\right|}^{k}=\operatorname{MCS}(\mathrm{CQI}=1)\right]^{T}$.

(2) Select the UE with the largest amount of required RBs in $g_{k}$. If there is a tie, randomly select one. If the selected UE's MCS level is MCS(CQI = 15) or the required amount of TTIs is one, then go to step (3); if not, increase its CQI by one, set $\wp=\wp+1$, calculate $g_{k}$ 's new total amount of required RBs and total energy consumption, and record this candidate MCS configuration pattern $x_{\wp}^{k}$. Then, repeat step (2).

(3) Check the recorded MCS configuration patterns in steps (1) and (2). If there is more than 1 pattern requiring the same amount of $\mathrm{RBs}$, only reserve the one with the least total energy consumption.

By the above listing method, for each group $g_{k}$, the total number of feasible MCS configuration patterns, $\mathfrak{\Im}_{k}$, would be less than $15 \times\left|g_{k}\right|$ and even less, which is a significant improvement compared to $15^{\left|g_{k}\right|}$.

Theorem 2. For each concurrent transmission group $g_{k}$, the amount of feasible MCS configuration patterns $\mathfrak{\Im}_{k} \leq 15 \times\left|g_{k}\right|$.

\section{Complexity Analysis}

In this section, we analyze the complexity of the proposed method. Assume there are $M$ RNs and $N$ UE items and the worst case analysis will be illustrated. The whole method can be divided into two parts. The first part includes the uplink path selection and grouping algorithm, while the second part deals with MCS level reselection. The two parts 
will be analyzed separately first. In the end, we sum up the complexities of the two parts.

Part I Analysis. For each UE item, calculate $M+1$ channel conditions for $M$ RNs and the eNB and then select the best one from $M+1$ candidate base stations which will cost

$$
O(2 \times N(M+1)) \sim O(N M)
$$

For the spatial reuse group formulation, we first calculate the weight of each UE item and this costs $O(N)$. Then, select one UE item with the maximum weight from each $\mathrm{RN}_{j}, j=$ $0, \ldots, M$. Assume that for each $\mathrm{RN}_{j}, j=0, \ldots, M$, there are $N_{j}$ UE items connecting to it and $N_{0}+\cdots+N_{M}=N$. So, selecting UE items to form group costs

$$
O\left(N_{1}\right)+\cdots+O\left(N_{M+1}\right) \sim O(N) .
$$

Calculate the transmit powers of UE items in a group cost at most

$$
O\left((M+1)^{2}\right) \sim O\left(M^{2}\right)
$$

Calculate $E_{k}^{\text {th }}$ and determine whether a group shall exclude UE items or not which needs

$$
O(M+1) \sim O(M) .
$$

If the result is to exclude some UE (UE items) from the group, execute the exclusion algorithm. In the exclusion algorithm, we first find out the UE which has to be excluded. Calculate the transmit interference and received interference of a UE cost $O(M+M)$. Then, for a group of UE items, the total complexity is

$$
O((M+1) \times(M+M)) \sim O\left(M^{2}\right) .
$$

To find out the UE with the maximum total interference costs

$$
O(M+1) \sim O(M) .
$$

After exclusion, we have to update the transmit powers of UE items in the group and check whether the exclusion is needed or not. Consider the worst case that the exclusion will be repeatedly executed until there is only one UE item remaining in the group. Then, the complexity for finding a spatial reuse group is

$$
\begin{aligned}
& O(M) \times\left(O\left(M^{2}\right)+O(M)+O\left(M^{2}\right)+O(M)\right) \\
& \quad \sim O\left(M^{3}\right)
\end{aligned}
$$

where $\left(O\left(M^{2}\right)+O(M)+O\left(M^{2}\right)+O(M)\right)$ is the summation of (14), (15), (16), and (17). In a worst case, we will form at most $\mathrm{N}$ single member groups and the complexity is

$$
\begin{aligned}
& \left(O(N)+O(N)+O\left(M^{3}\right)\right) \times O(N) \\
& \sim O\left(N^{2}\right)+O\left(N M^{3}\right) .
\end{aligned}
$$

The first $O(N)$ is the complexity of updating weights after each time grouping a group. The second $O(N)$ is the complexity of selecting $M+1$ UE items out of $N$ UE items to form a group. The third $O\left(M^{3}\right)$ is the complexity of (18).

Therefore, the complexity of Part I is

$$
O(N M)+O\left(N^{2}\right)+O\left(N M^{3}\right)
$$

by summing (12) and (19) up.

Part II Analysis. For each group $g_{k}, k=1, \ldots, K$, at most $15 \times\left|g_{k}\right|$ CQI combinations have to be listed. For each group, this costs $O\left(15\left|g_{k}\right|\right)$. Because $\left|g_{1}\right|+\left|g_{2}\right|+\cdots+\left|g_{K}\right|=N$, the total complexity of listing all CQI combinations can be expressed as

$$
O(15 N) \sim O(N)
$$

Then, calculate the penalty table for each group. This involves the transmit power and consumed energy calculation. So the complexity of calculating the penalty table for a group $g_{k}$ is

$$
O\left(15\left|g_{k}\right|\right) \times O\left(15\left|g_{k}\right|^{2}\right) \sim O\left(\left|g_{k}\right|^{3}\right) \text {. }
$$

The upper bound of (22) is $O\left(M^{3}\right)$ when the group size $\left|g_{k}\right|=$ $M+1$. For $K$ groups, the total complexity is $O(K) \times O\left(\left|g_{k}\right|^{3}\right)$. Selecting the minimum penalty costs $O(N)$. For the selected group, we enhance the CQI and then update the penalty table of the selected group. The updating cost is $O\left(15\left|g_{k}\right|\right) \sim$ $O\left(\left|g_{k}\right|\right)$.

Above MCS level reselection will be repeated until the total number of required resources of UE items is less than or equal to the total system bandwidth. For the worst case, all UE items have to be upgraded to the highest level of CQI to meet the requirement. In this case, the preceding steps must be executed $15 \mathrm{~N}$ times. An alternative way to evaluate the execution time is as below. Assume that the total number of required resources is $\sum_{\forall i} R_{i}$, where $R_{i}$ is the largest amount of required TTIs of group $i$ when CQI $=1$ is used. For each time we upgrade the CQI of a group, at least 1 TTI can be reduced from the number of total required resources. So, MCS reselection must be executed at most $\left(\sum_{\forall i} R_{i}-\left(F_{\mathrm{B}}+F_{\mathrm{nB}}\right)\right)$ times. Therefore, the execution time of MCS reselection can be expressed as

$$
L=\min \left\{O(15 N),\left(\sum_{\forall i} R_{i}-\left(F_{\mathrm{B}}+F_{\mathrm{nB}}\right)\right)\right\} .
$$

So the total complexity of Part II is

$$
\begin{aligned}
& O(N)+O(K) \times O\left(\left|g_{k}\right|^{3}\right)+L \times\left(O(N)+O\left(\left|g_{k}\right|\right)\right) \\
& \quad \leq O(N)+O\left(N M^{2}\right)+L \times(O(N)) \\
& \quad \leq O(N)+O\left(N M^{2}\right)+O(15 N) \times(O(N)) \\
& \quad \sim O\left(N^{2}\right)+O\left(N M^{2}\right) .
\end{aligned}
$$

Combining Part I (20) and Part II (24), the total complexity is

$$
O\left(N^{2}\right)+O\left(N M^{3}\right)
$$


TABLE 3: The parameters in our simulation.

\begin{tabular}{|c|c|}
\hline Parameter & Value \\
\hline Channel bandwidth & $10 \mathrm{MHz}$ \\
\hline Intersite distance (ISD) & $500 \mathrm{~m}$ (Case 1$)$ \\
\hline \multirow{10}{*}{ Channel model } & $L(R)=P L_{\mathrm{LOS}}(R) \times \operatorname{Prob}(R)+(1-\operatorname{Prob}(R)) \times P L_{N \mathrm{LOS}}(R)$ \\
\hline & $R$ : distance in kilometers \\
\hline & eNB-UE \\
\hline & $P L_{\mathrm{LOS}}(R)=103.4+24.2 \log 10(R)$ \\
\hline & $P L_{N L O S}(R)=131.1+42.8 \log 10(R)$ \\
\hline & $\operatorname{Prob}(R)=\min (0.018 / R, 1) \times(1-\exp (-R / 0.063))+\exp (-R / 0.063)$ \\
\hline & RN-UE \\
\hline & $P L_{\mathrm{LOS}}(R)=103.8+20.9 \log 10(R)$ \\
\hline & $P L_{N L O S}(R)=145.4+37.5 \log 10(R)$ \\
\hline & $\operatorname{Prob}(R)=0.5-\min (0.5,5 \exp (-0.156 / R))+\min (0.5,5 \exp (-R / 0.03))$ \\
\hline eNB maximum transmit power & $30 \mathrm{dBm}$ \\
\hline eNB maximum antenna gain & $14 \mathrm{dBi}$ \\
\hline RN maximum transmit power & $30 \mathrm{dBm}$ \\
\hline RN maximum antenna gain & $5 \mathrm{dBi}$ \\
\hline UE maximum transmit power & $23 \mathrm{dBm}$ \\
\hline UE maximum antenna gain & $0 \mathrm{dBi}$ \\
\hline Thermal noise & $-174 \mathrm{dBm}$ \\
\hline \multirow{6}{*}{ Traffic } & Case 1: \\
\hline & Audio: $4-25 \mathrm{kbits} / \mathrm{s}$ \\
\hline & Video: $32-384$ kbits/s \\
\hline & Data: $60-384 \mathrm{kbits} / \mathrm{s}$ \\
\hline & Case 2: \\
\hline & Audio: 4-25 kbits/s \\
\hline
\end{tabular}

Consider that $M$ is usually a finite constant, so the complexity of the proposed method is $O\left(N^{2}\right)$.

\section{Simulation Results}

We develop a simulator in MATLAB to verify the effectiveness of our heuristics. The system parameters in the simulation are listed in Table 3 [3]. We consider three types of traffic: audio, video, and data [25]. Two traffic cases are applied in the simulation. Traffic Case 1 is mixed traffic, where each UE item executes an audio, video, or data flow with equal probability. On the other hand, Traffic Case 2 only contains audio traffic. The network contains one eNB and six RNs $(M=6)$. RNs are uniformly deployed inside the $2 / 3$ coverage range of the eNB to get the best performance gain. In default, we set the factors $\alpha, \beta$, and $\gamma$ to 1 to get the best performance and adopt TDD mode uplink-downlink configuration 1; that is, there are 4 uplink subframes per frame. The ratio of uplink backhaul subframe and uplink nonbackhaul subframe is $1: 3$. We compare the performances of four methods: (1) OEA (Opportunistic and Efficient RB Allocation) [14], (2) EPAR (Equal Power Allocation with Refinement) [17], (3) our proposed scheme without relay nodes, and (4) our proposed scheme.

Figures 7(a) and 7(b) evaluate the total energy consumption of UE items under different number of UE items
$(N)$ when Traffic Cases 1 and 2 are applied, respectively. Both figures show that as $N$ increases, the total amount of energy consumption of UE items increases for all methods. OEA consumes the most energy because UE items always connect to the eNB and select the most efficient MCS for transmission. EPAR performs better than OEA because celledge UE items can choose to connect with RNs instead of the eNB and this reduces the energy consumption. With our energy-saving resource allocation method, the proposed scheme (w/o relay) performs the second. Results show that our proposed scheme performs the best in all methods. This means that spatial reuse and RNs do help the reduction of total energy consumption of UE items. In Figure 7(b), our heuristics still performs the best compared to the other 3 methods. Obviously, the spatial reuse and energy-saving resource allocation do help to conserve UE items' energy. One interesting thing is that when $N$ is large, EPAR and the proposed scheme (w/o relay) consume almost the same energy. This is because relay improves the SINR of cell-edge users, thus reducing the energy consumption of edge users.

Figures $8(\mathrm{a})$ and $8(\mathrm{~b})$ evaluate the bandwidth utilization under different number of UE items for Traffic Cases 1 and 2, respectively. OEA and EPAR always pursue the most efficient MCS. When the traffic load is light, the bandwidth utilization hurts and results in much idle bandwidth. On the other hand, the proposed scheme and proposed scheme w/o relay get the 


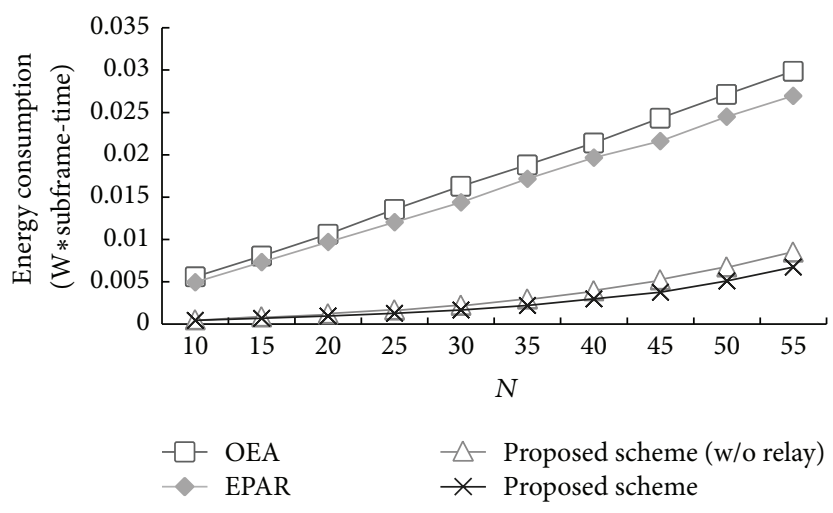

(a) Traffic Case 1

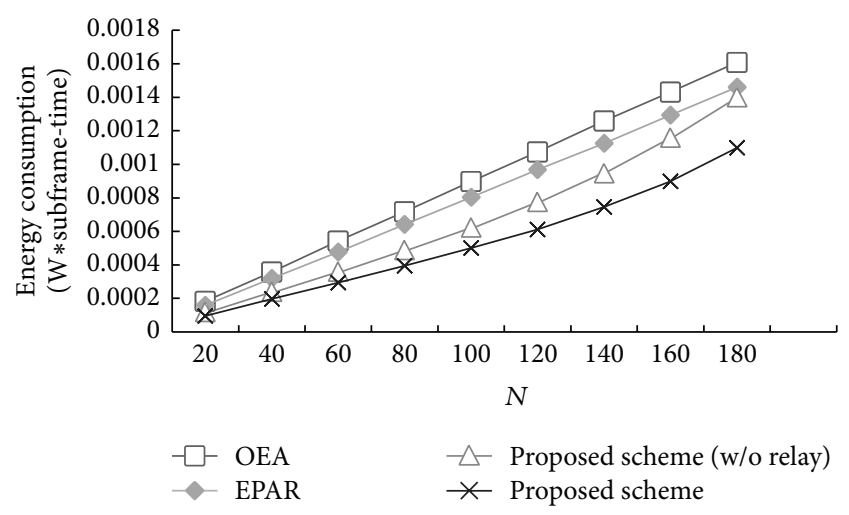

(b) Traffic Case 2

Figure 7: The impact of $N$ on the total energy consumption $(M=6)$.

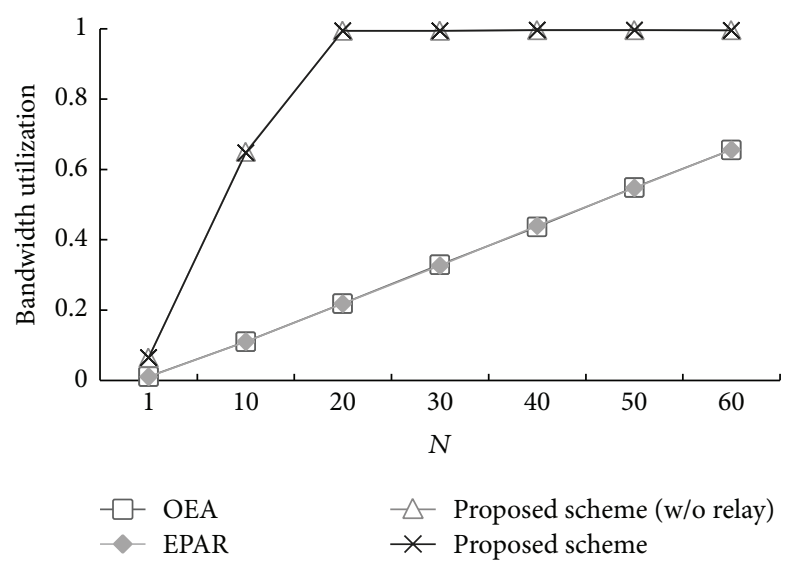

(a) Traffic Case 1

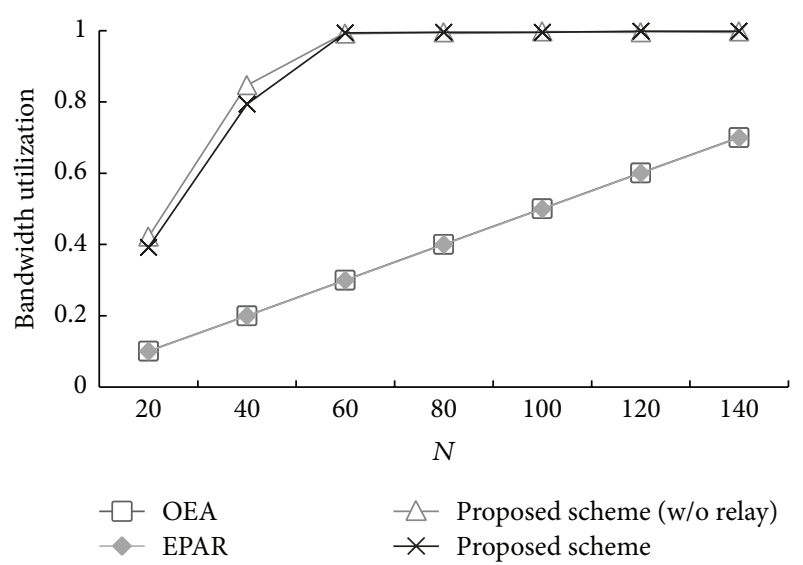

(b) Traffic Case 2

Figure 8: The impact of $N$ on the bandwidth utilization $(M=6)$.

best bandwidth utilization in all four methods. The results show that our proposed methods can improve the bandwidth utilization and save more energy for UE items.

Figures 9(a) and 9(b) show the impact of $N$ on the throughput for Traffic Cases 1 and 2, respectively. As shown in the figures, as $N$ increases, the throughput of all schemes increases. We can see that the proposed methods can guarantee all the traffic demand being served like OEA and EPAR. This means that when the network load is light, our schemes can well utilize the idle bandwidth to reduce UE items' uplink transmit power. On the contrary, when the network load is heavy, our schemes will select efficient MCS for UE items to reduce each of their required physical radio resources such that the admitted data rates of UE items can still be satisfied. So, our proposed schemes can not only provide similar throughput like OEA and EPAR, but also save UE items' energy.

Figure 10 shows the average extra data transmission delay of the proposed schemes and EPAR against OEA. Compared to OEA, EPAR causes a longer delay because RUEs have to deliver their data to the eNB via RNs. But in OEA, UE items directly transmit their data to the eNB. The proposed schemes have a longer delay compared to both OEA and EPAR because they utilize more physical resources to deliver data, thus resulting in more extra data packet buffering delay. As $N$ increases, the result shows that the extra delay does not always increase (when $N \leq 20$ ) but decreases after $N$ is more than 20. This is because OEA needs more time to deliver users' data when traffic load is heavy, but the proposed schemes consume the same time and upgrade UE items' MCS level instead. Our proposed methods slightly increase the delay of data transmission, but the average extra delay is no more than $5 \mathrm{~ms}$ as shown in Figure 10. It should be acceptable.

In Figure 11, we discuss the effect of subframe configuration on the total energy consumption of UE items. In the TDD mode LTE-A relay network, it supports four kinds of uplink nonbackhaul and backhaul subframe configurations: (1) 1 uplink nonbackhaul subframe and 1 uplink backhaul subframe per frame (1a:1b), (2) 2 uplink nonbackhaul subframes and 1 uplink backhaul subframe per frame (2a:1b), (3) 2 uplink nonbackhaul subframes and 2 uplink backhaul subframes per frame (2a:2b), and (4) 3 uplink nonbackhaul subframes and 1 uplink backhaul subframe per frame ( $3 a: 1 b)$. As shown in Figure 11, no matter which subframe configurations 


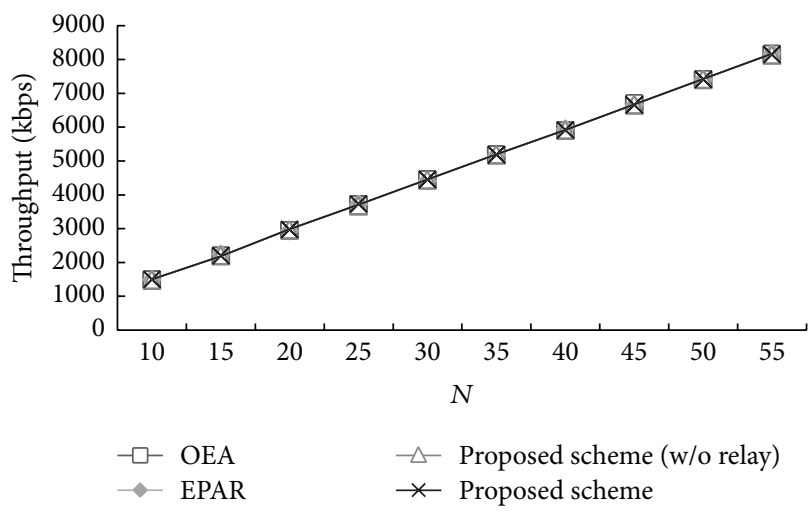

(a) Traffic Case 1

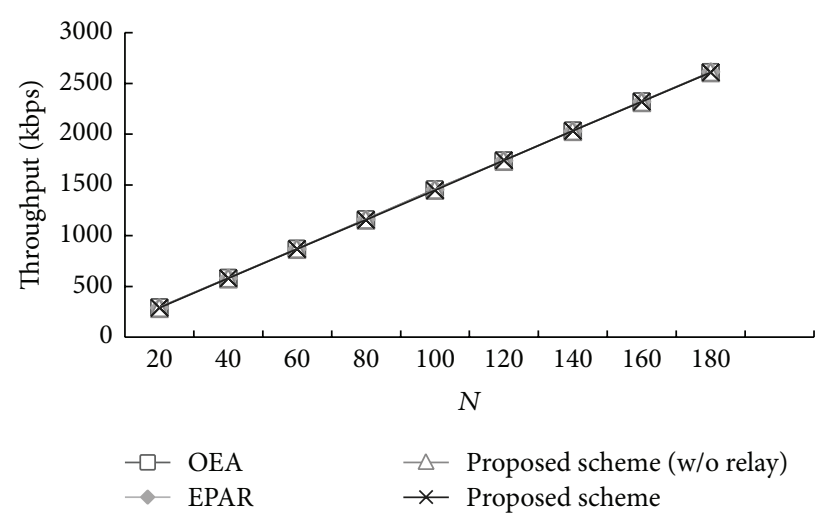

(b) Traffic Case 2

FIgURE 9: The impact of $N$ on the throughput $(M=6)$.

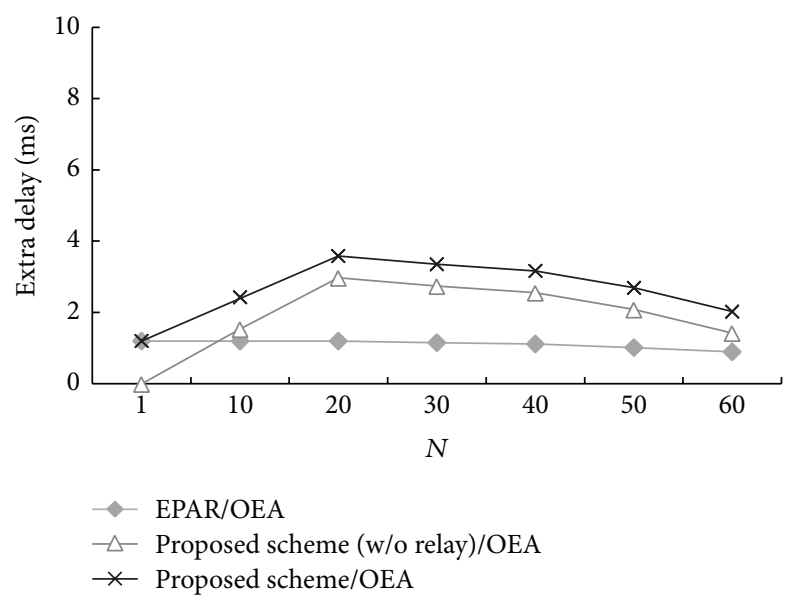

FIGURE 10: The average extra data transmission delay of all schemes compared to OEA $(M=6$, Traffic Case 1$)$.

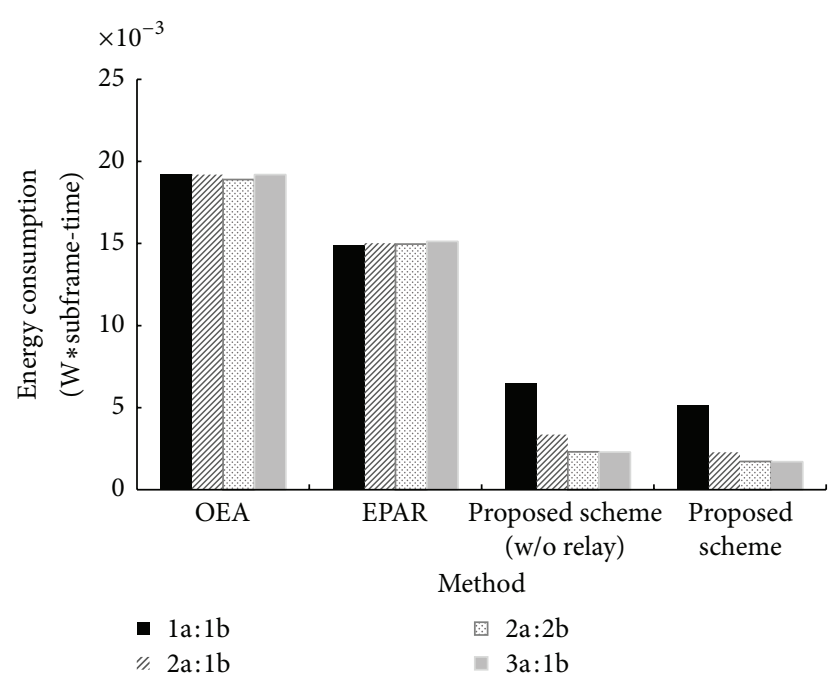

FIgURE 11: The impact of subframe configurations on the total energy consumption ( $N=35$ and $M=6$, Traffic Case 1$)$. are used, our method always gets the best power saving in all schemes. For OEA and EPAR, the performances are almost the same for all four kinds of subframe configurations. This is because they always use the most efficient MCS no matter whether the uplink radio resources are many or few. The proposed schemes reduce the energy consumption of UE items by well utilizing the idle radio resource. Therefore, the result shows that the total energy consumption of UE items decreases in the proposed methods as the number of uplink subframe increases (number of uplink subframes per frame is increased from $2(1 \mathrm{a}: 1 \mathrm{~b})$ to $4(2 \mathrm{a}: 2 \mathrm{~b}$ or $3 \mathrm{a}: 1 \mathrm{~b}))$. When the network has more radio resources, UE items can choose to use lower level of MCS to transmit data and save energy. Comparing subframe configurations $2 \mathrm{a}: 2 \mathrm{~b}$ and $3 a: 1 b$, Figure 11 shows that the latter can conserve more energy. The higher number of nonbackhaul subframes means there are more resources that can be used by MUEs and RUEs, but the backhaul subframe can only be utilized by MUEs. Obviously, the former provides more flexibility. This is why subframe configuration $3 \mathrm{a}: 1 \mathrm{~b}$ conducts better energy saving than that of $2 \mathrm{a}: 2 \mathrm{~b}$.

In Figure 12, Traffic Case 2 is applied to evaluate the effect of subframe configuration on the total energy consumption of UE items. The proposed scheme performs the best in all 4 schemes. Compared to the previous experiment as shown in Figure 11, Figure 12 shows that the performance differences among all four schemes become smaller. This is because, in Traffic Case 2, the data size is small compared to the number of radio resources provided in one single TTI; then in our implementation, OEA and EPAR will automatically apply a low level MCS to fill up the whole space of assigned radio resource. This is why we see a closer performance among the four schemes.

Then, Figure 13 evaluates the total energy consumption of $\mathrm{UE}$ items over different ratio of $\beta / \alpha$. Figure 13 presents that as $\beta / \alpha$ increases, the total energy consumption decreases when $\beta / \alpha \leq 1$. This means that factor 1 (path loss factor) and factor 2 (data size factor) of (8) have equal importance for weight $W_{i}$. When choosing the reuse group, the distance between a UE item and the connected $\mathrm{RN}$ and the size of the data request are both significant factors for energy conservation. 


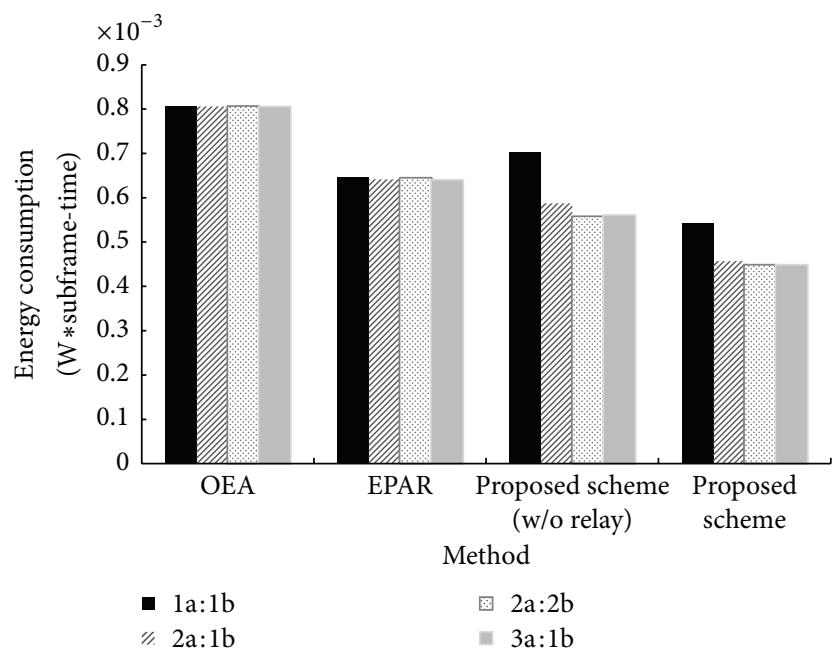

Figure 12: The impact of subframe configurations on the total energy consumption ( $N=90$ and $M=6$, Traffic Case 2).

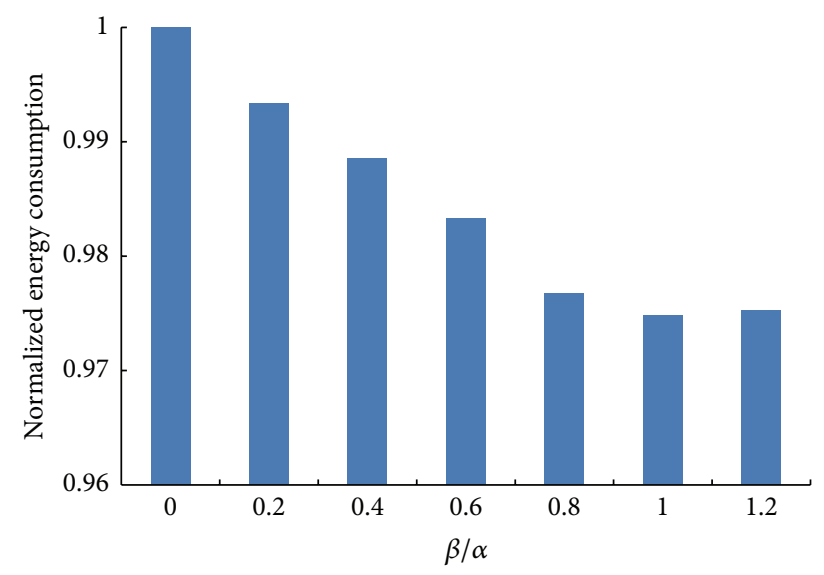

FIGURE 13: The impact of $\beta / \alpha$ on the total energy consumption $(N=$ 40 and $M=3$ ).

Figure 14 shows the total energy consumption over different $\gamma$, where we set $\alpha=\beta=1$. It can be seen that the total energy consumption performs the worst when $\gamma=0$. This means that $\gamma$ does help the selection of spatial reuse groups. With a nonzero $\gamma$, we can filter out unsuitable UE items when forming reuse groups.

\section{Conclusion}

In this paper, we investigate the energy conservation issue of the uplink path, uplink radio resource, MCS, and mobile device transmit power allocation in LTE-A relay networks. We have proposed heuristics to conserve UE items' energy by exploiting RNs, MCS, BER, transmit power, and spatial reuse. To save energy, the key factors are how to determine the most energy-saving MCS of UE items and how to select interference-free spatial reuse groups. To find the best settings, we have defined the weight and penalty functions for evaluation. Simulation results show that our scheme can

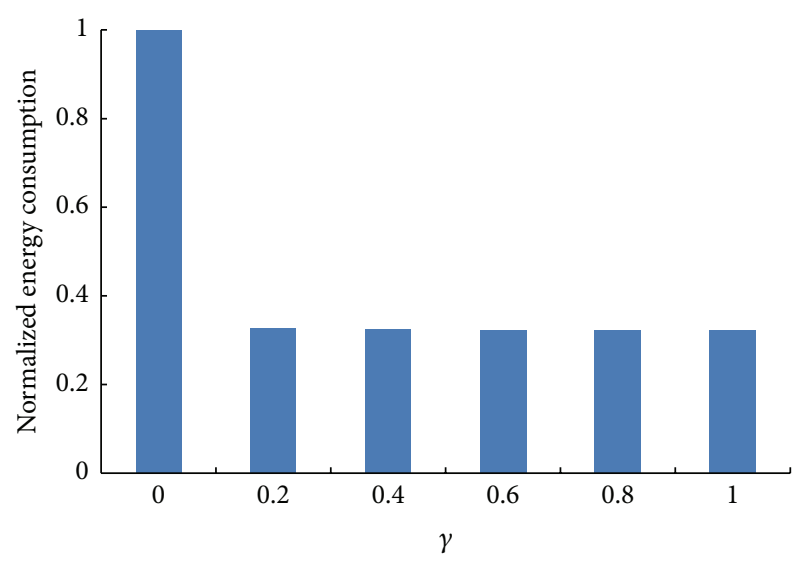

FIGURE 14: The impact of $\gamma$ on the total energy consumption where $\beta=\alpha=1(N=50$ and $M=3)$.

significantly reduce the total energy consumption of UE items compared to other schemes and has good bandwidth utilization. Compared with OEA and EPAR schemes, our proposed energy-saving resource allocation method will slightly increase the delay of data, but the extra delay is less than one frame (no more than $10 \mathrm{~ms}$ ). Users' required QoS, $\mathrm{BER}$, and throughput can all be guaranteed.

\section{Notations}

$N$ :

$M:$

$F_{\mathrm{B}}$ :

$F_{\mathrm{nB}}$ :

$P_{i}:$

$E_{i}$ :

$\delta_{i}$ :

$T_{i}^{\mathrm{UE} \_\mathrm{RN}}:$

$T_{i}^{\mathrm{RN} \_\mathrm{BS}}$ :

$W_{i}:$

$g_{k}:$

$E_{k}^{\text {th }}$ :

$E_{x}^{k}$ :

$A_{x}^{k}$

$I_{m, n}^{t}$

$I_{m, n}^{r}$ :

$d_{i, j}:$

$t_{i}$ :

rate $(\mathrm{CQI}=k)$ : The code rate when using CQI $k$ (in bits/TTI)

Number of UE items

Number of RNs

The total amount of TTIs for uplink backhaul subframes per frame The total amount of TTIs for uplink nonbackhaul subframes per frame The transmit power of $\mathrm{UE}_{i}$ The energy cost of $\mathrm{UE}_{i}$ The uplink traffic demand of $\mathrm{UE}_{i}$ per frame

The amount of required TTIs for $\mathrm{UE}_{i}$ to deliver data to its connected RN The amount of required TTIs for $\mathrm{UE}_{i}$ 's connected $\mathrm{RN}$ to deliver data to the eNB The weight of $\mathrm{UE}_{i}$ The concurrent transmission group $k$ Energy threshold of $g_{k}$

Total amount of energy consumption of $g_{k}$ when using CQI $x$

Total amount of required uplink TTIs for $g_{k}$ when using CQI $x$

Transmit interference for the transmission pair $\left(\mathrm{UE}_{m}, \mathrm{RN}_{n}\right)$ Received interference for the transmission pair $\left(\mathrm{UE}_{m}, \mathrm{RN}_{n}\right)$ The distance between $\mathrm{UE}_{i}$ and $\mathrm{RN}_{j}$ Number of exclusion times of $\mathrm{UE}_{i}$ 


$\begin{array}{ll}\text { MCS }(C Q I=k): & \text { The corresponding MCS when using } \\ & \text { CQI } k \\ B: & \text { Effective bandwidth (in Hz) } \\ N_{0}: & \text { Thermal noise } \\ G_{i}: & \text { Antenna gain of node } i \\ P_{i, j}: & \text { The received power from transmitter } i \\ & \text { to receiver } j \\ I_{i, j}: & \text { The interference to receiver } j \text { from } \\ & \text { transmitters other than } i\end{array}$

\section{Competing Interests}

The authors declare that they have no competing interests.

\section{Acknowledgments}

This research is sponsored by MOST 104-2221-E-024-005.

\section{References}

[1] D. Astély, E. Dahlman, A. Furuskär, Y. Jading, M. Lindström, and S. Parkvall, "LTE: the evolution of mobile broadband," IEEE Communications Magazine, vol. 47, no. 4, pp. 44-51, 2009.

[2] 3GPP TR 36.913 v12.0.0, "Requirements for further advancements for E-UTRA (LTE-Advanced)," September 2014.

[3] 3GPP, "Further advancements for E-UTRA physical layer aspects," 3GPP TR 36.814 v9.0.0, 2010.

[4] P. K. Dalela, A. Nayak, V. Tyagi, and K. Sridhara, "Analysis of spectrum utilization for existing cellular technologies in context to cognitive radio," in Proceedings of the 2 nd International Conference on Computer and Communication Technology (ICCCT '11), pp. 585-588, Allahabad, India, September 2011.

[5] I. C. Wong, O. Oteri, and W. McCoy, "Optimal resource allocation in uplink SC-FDMA systems," IEEE Transactions on Wireless Communications, vol. 8, no. 5, pp. 2161-2165, 2009.

[6] S.-B. Lee, I. Pefkianakis, A. Meyerson, S. Xu, and S. Lu, "Proportional fair frequency-domain packet scheduling for 3GPP LTE uplink," in Proceedings of the 28th Conference on Computer Communications (IEEE INFOCOM '09), pp. 26112615, IEEE, Rio de Janeiro, Brazil, April 2009.

[7] Z. Ma, W. Xiang, H. Long, and W. Wang, "Proportional fair resource partition for LTE-advanced networks with type I relay nodes," in Proceedings of the IEEE International Conference on Communications (ICC '11), pp. 1-5, Kyoto, Japan, June 2011.

[8] G. Liebl, T. M. de Moraes, A. Soysal, and E. Seidel, "Fair resource allocation for the relay backhaul link in LTE-Advanced," in Proceedings of the EEE Wireless Communications and Networking Conference (WCNC '12), pp. 1196-1201, Shanghai, China, April 2012.

[9] J.-P. Yoon, W.-J. Kim, J.-Y. Baek, and Y.-J. Suh, "Efficient uplink resource allocation for power saving in IEEE 802.16 OFDMA systems," in Proceedings of the IEEE 67th Vehicular Technology Conference (VTC Spring '08), pp. 2167-2171, Singapore, May 2008.

[10] J.-M. Liang, Y.-C. Wang, J.-J. Chen, J.-H. Liu, and Y.-C. Tseng, "Energy-efficient uplink resource allocation for IEEE 802.16j transparent-relay networks," Computer Networks, vol. 55, no. 16, pp. 3705-3720, 2011.
[11] M. Lauridsen, A. R. Jensen, and P. Mogensen, "Reducing LTE uplink transmission energy by allocating resources," in Proceedings of the IEEE 74th Vehicular Technology Conference (VTC Fall '11), pp. 1-5, September 2011.

[12] Y. Zou, J. Zhu, and B. Y. Zheng, "Energy efficiency of network cooperation for cellular uplink transmissions," in Proceedings of the IEEE International Conference on Communications (ICC '13), pp. 4394-4398, IEEE, Budapest, Hungary, June 2013.

[13] M. Kalil, A. Shami, and A. Al-Dweik, "QoS-aware powerefficient scheduler for LTE uplink," IEEE Transactions on Mobile Computing, vol. 14, no. 8, pp. 1672-1685, 2015.

[14] F. Z. Kaddour, E. Vivier, L. Mroueh, M. Pischella, and P. Martins, "Green opportunistic and efficient resource block allocation algorithm for LTE uplink networks," IEEE Transactions on Vehicular Technology, vol. 64, no. 10, pp. 4537-4550, 2015.

[15] R. Imran, M. Shukair, N. Zorba, O. Kubbar, and C. Verikoukis, "A novel energy saving MIMO mechanism in LTE systems," in Proceedings of the IEEE International Conference on Communications (ICC '13), pp. 2449-2453, Budapest, Hungary, June 2013.

[16] P.-C. Lin, R.-G. Cheng, and Y.-J. Chang, "A dynamic flow control algorithm for LTE-advanced relay networks," IEEE Transactions on Vehicular Technology, vol. 63, no. 1, pp. 334-343, 2014.

[17] M. S. Alam, J. W. Mark, and X. S. Shen, "Relay selection and resource allocation for multi-user cooperative OFDMA networks," IEEE Transactions on Wireless Communications, vol. 12, no. 5, pp. 2193-2205, 2013.

[18] N. Krishnan, R. D. Yates, N. B. Mandayam, and J. S. Panchal, "Bandwidth sharing for relaying in cellular systems," IEEE Transactions on Wireless Communications, vol. 11, no. 1, pp. 117$129,2012$.

[19] T. H. Cormen, C. E. Leiserson, R. L. Rivest, and C. Stein, Introduction to Algorithms, MIT Press, Cambridge, Mass, USA, 3rd edition, 2009.

[20] 3GPP, "Physical channels and modulation," 3GPP TS 36.211 v13.0.0, 2015.

[21] 3GPP TS 36.216 v13.0.0, "Physical layer for relaying operation," December 2015.

[22] 3GPP, "E-UTRA; physical layer procedures," 3GPP TS 36.213 v12.0.0, 2013

[23] J. Blumenstein, J. Ikuno, J. C. Prokopec, and M. Rupp, "Simulating the long term evolution uplink physical layer," in Proceedings of the ELMAR, pp. 141-144, IEEE, Zadar, Croatia, September 2011.

[24] H. Kellerer, U. Pferschy, and D. Pisinger, Knapsack Problems, Springer, Berlin, Germany, 2004.

[25] 3GPP, "Services and service capabilities," 3GPP TS 22.105 v10.0.0, 2011. 

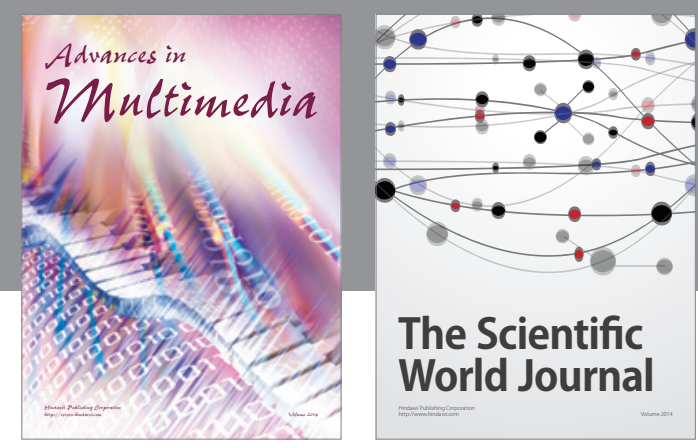

The Scientific World Journal
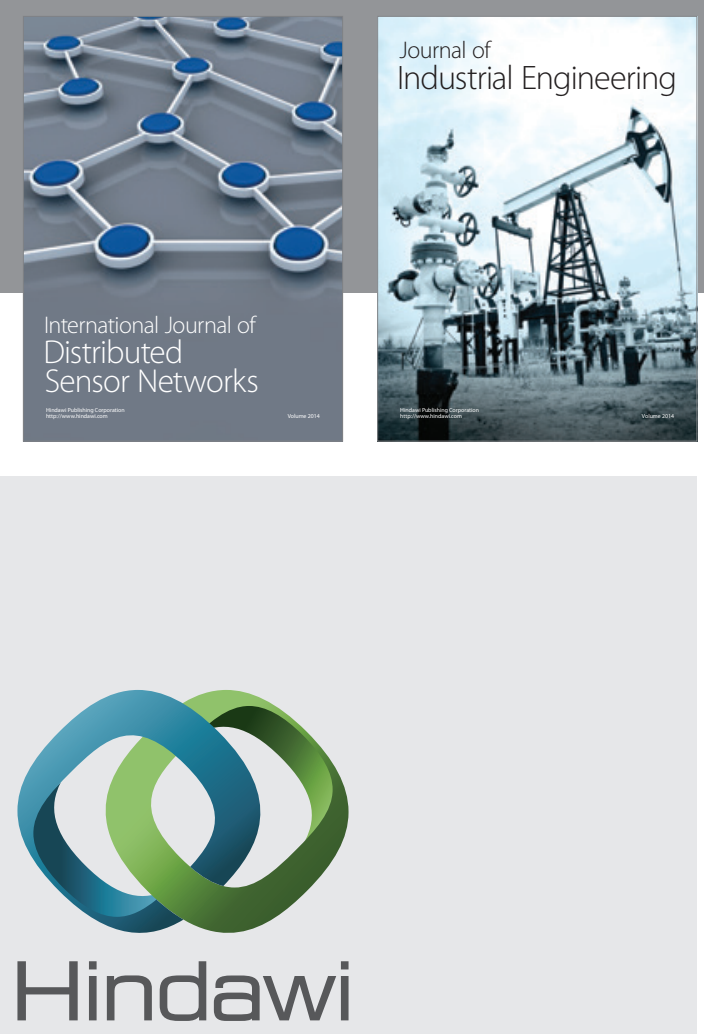

Submit your manuscripts at

http://www.hindawi.com

\section{Computer Networks} and Communications
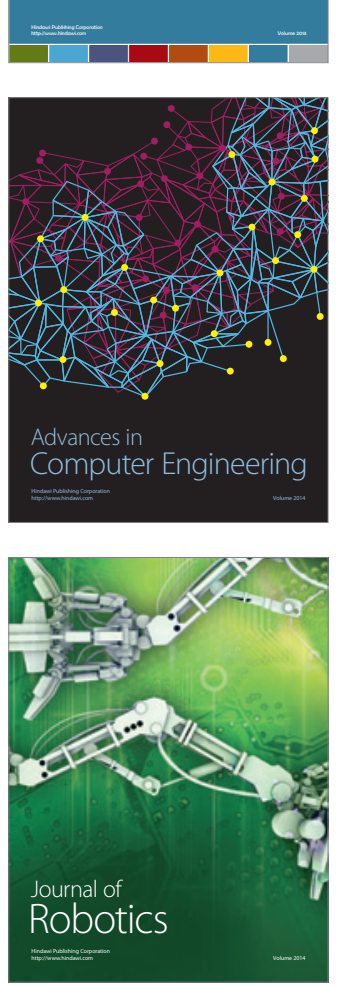
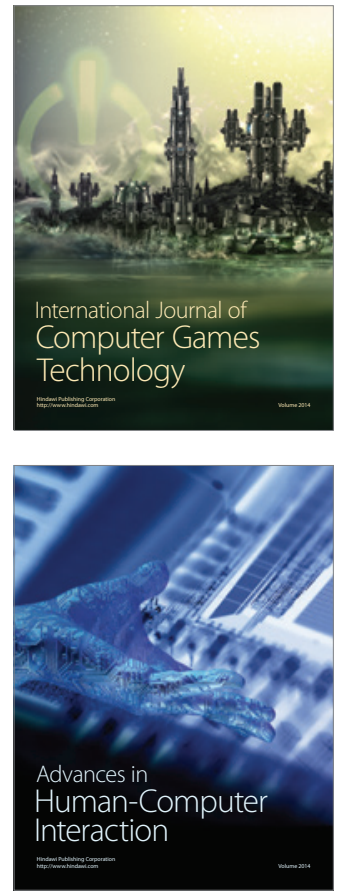
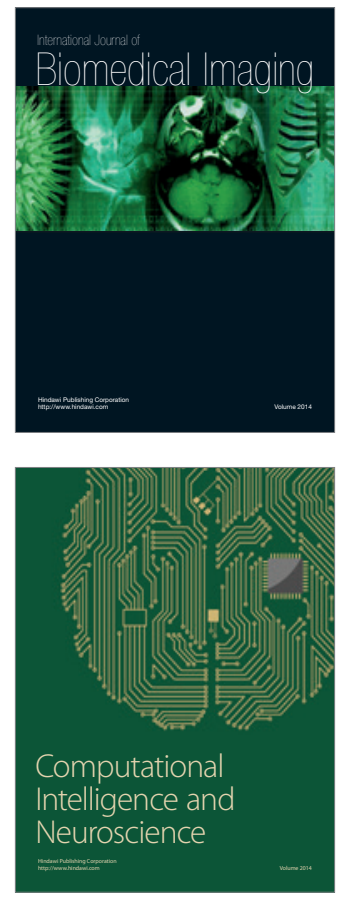
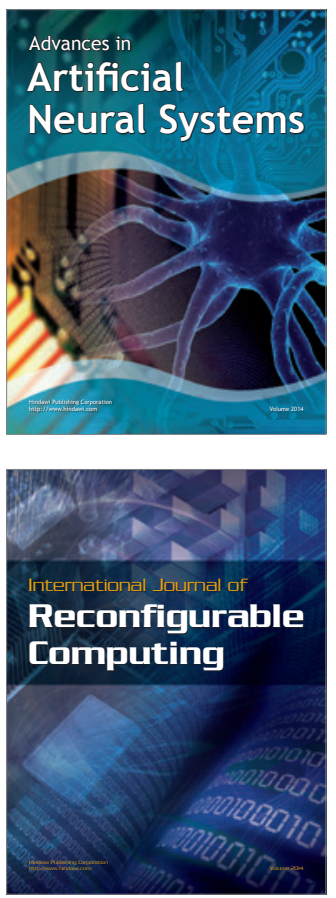
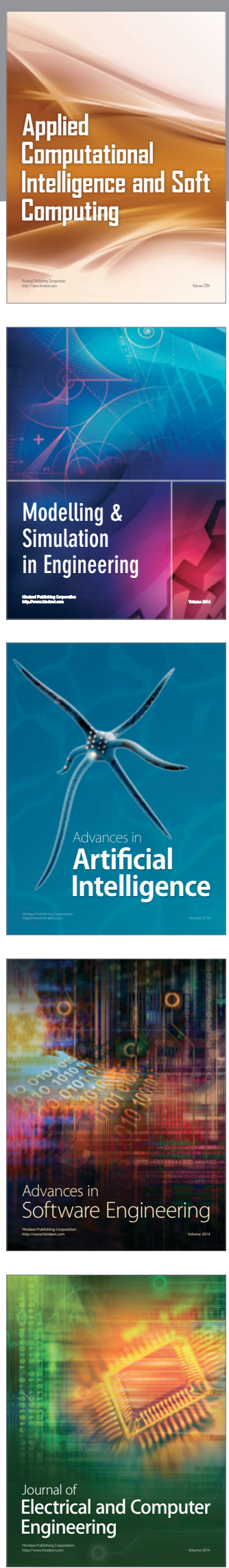